\title{
Averaging theorems in finite deformation plasticity
}

\author{
Sia Nemat-Nasser ${ }^{1}$ \\ Center of Excellence for Advanced Materials, Department of Applied Mechanics and Engineering Sciences, University of California, \\ San Diego, MC 0416, 9500 Gilman Drive, La Jolla, CA 92093-0416, USA
}

Received 27 January 1997; received in revised form 8 October 1998

\begin{abstract}
The transition from micro- to macro-variables of a representative volume element (RVE) of a finitely deformed aggregate (e.g., a composite or a polycrystal) is explored. A number of exact fundamental results on averaging techniques, valid at finite deformations and rotations of any arbitrary heterogeneous continuum, are obtained. These results depend on the choice of suitable kinematical and dynamical variables. For finite deformations, the deformation gradient and its rate, and the nominal stress and its rate, are optimally suited for the averaging purposes. A set of exact identities is presented in terms of these variables. An exact method for homogenization of an ellipsoidal inclusion in an unbounded finitely deformed homogeneous solid is presented, generalizing Eshelby's method for application to finite deformation problems. In terms of the nominal stress rate and the rate of change of the deformation gradient, measured relative to any arbitrary state, a general phase-transformation problem is considered, and the concepts of eigenvelocity gradient and eigenstress rate are introduced. It is shown that the velocity gradient (and hence the nominal stress rate for rate-independent models) in an ellipsoidal region within an unbounded uniform and uniformly deformed solid, remains uniform when this region undergoes a uniform phase transformation corresponding to a constant eigenvelocity gradient. The generalized Eshelby tensor and its conjugate are defined and used to obtain the field quantities in an ellipsoidal inclusion which is embedded in an unbounded, uniformly deformed medium, leading to exact expressions for the concentration tensors and a set of identities relating these quantities. The Green functions for the rate quantities measured from a finitely deformed state, are formulated, and their properties are discussed. The details for calculating the Green function, Eshelby's tensor and its conjugate, and the concentration tensors, are presented. The relation between strong ellipticity and Green's function is examined in connection with the problem of the possible loss of stability of a uniformly stressed homogeneous solid. Exact values of the average nominal stress rate and the average velocity gradient, taken over an ellipsoidal region in a finitely deformed unbounded homogeneous solid, are obtained when arbitrary (variable) eigenvelocity gradients or eigenstress rates are prescribed in any region within the ellipsoid. It is shown that many results for single- and double-inclusion problems in linear elasticity, also apply to the finite-deformation rate problems, provided suitable kinematical and dynamical variables are used. The problem of the double inclusion is considered and exact expressions are given for the average field quantities, taken over the region between the two ellipsoidal domains, one containing the other, when arbitrary eigenvelocity gradients are prescribed within an arbitrary region contained in the inner ellipsoid. These results are further generalized to obtain exact expressions for the average field quantities taken over each annulus of a nested sequence of ellipsoidal regions within a finitely (uniformly) deformed infinite solid, where a constant distinct eigenvelocity gradient is prescribed on each annulus. These results are new, and should provide effective tools for homogenization of multi-component composites at finite deformations and rotations. (c) 1999 Elsevier Science Ltd. All rights reserved.
\end{abstract}

\footnotetext{
${ }^{1}$ Tel.: +1 619534 4772; fax: +1 619534 2727; e-mail: sia@ceam.ucsd.edu
} 


\section{Introduction}

For linearly elastic heterogeneous solids, exact averaging theorems and several homogenization models are developed in detail by Nemat-Nasser and Hori (1993), starting with the pioneering works of Hill (1952, 1965a, b), Kröner (1958, 1977, 1978), Eshelby (1957), Hashin (1964, 1965), Budiansky (1965), Walpole (1966a, b), and Willis $(1977,1981)$. There are several basic contributions that can be used to develop a similarly comprehensive treatment in the general area of homogenization of finitely deformed heterogeneous solids; Hill (1968, 1970, 1972, 1984), Rice (1971), Havner (1982), and Iwakuma and Nemat-Nasser (1984). This is the main objective of the present contribution which seeks to address fundamental issues relating to rigorous treatment of transition from micro- to macro-variables of a representative volume element of a finitely deformed aggregate, e.g., a composite or a polycrystal. A number of exact fundamental results on averaging techniques, valid at finite deformations and rotations of any arbitrary heterogeneous continuum, are outlined. These results depend on the choice of suitable kinematical and dynamical variables. For finite deformations, the deformation gradient and its rate, and the nominal stress and its rate, are optimally suited for the averaging purposes. A set of exact identities are presented in terms of these variables.

An exact method for homogenization of an ellipsoidal inclusion in an unbounded finitely deformed homogeneous solid is presented, generalizing Eshelby's method for application to finite deformation problems. In terms of the nominal stress rate and the rate of change of the deformation gradient, measured relative to any arbitrary state, a general phase-transformation problem is considered, and the concepts of eigenvelocity gradient and eigenstress rate are introduced. The generalized Eshelby tensor and its conjugate are defined and used to obtain the field quantities in an ellipsoidal inclusion, leading to exact expressions for the concentration tensors and a set of identities relating these quantities.

The Green functions for the rate quantities measured from a finitely deformed state, are formulated, and their properties are discussed. The details for calculating the Green function, Eshelby's tensor and its conjugate, and the concentration tensors, are presented. The relation between strong ellipticity and Green's function is examined in connection with the problem of the possible loss of stability of a uniformly stressed homogeneous solid, making contact with fundamental works on necessary conditions for the formation of discontinuities in finitely and homogeneously deformed solids; Hadamard (1903), Thomas (1956, 1958), Hill (1961, 1962), and Rice (1977).

Exact values of the average nominal stress rate and the average velocity gradient, taken over an ellipsoidal region in a finitely deformed unbounded homogeneous solid, are obtained when arbitrary (variable) eigenvelocity gradients or eigenstress rates are prescribed in any region within the ellipsoid. It is shown that many results for single- and double-inclusion problems in linear elasticity, also apply to the finite-deformation rate problems, provided suitable kinematical and dynamical variables are used. Then, it is shown that the velocity gradient (and hence the nominal stress rate for rate-independent models) in an ellipsoidal region within an unbounded uniform and uniformly deformed solid, remains uniform when this region undergoes a uniform phase transformation corresponding to a constant eigenvelocity gradient. The problem of the double inclusion is considered and exact expressions are given for the average field quantities, taken over the region between the two ellipsoidal domains, one containing the other, when arbitrary eigenvelocity gradients are prescribed within an arbitrary region contained in the inner ellipsoid. This generalizes to the fully nonlinear, finitely deformed, elastoplastic case, the Tanaka and Mori (1972) result and the double inclusion result of Nemat-Nasser and Hori (1993), and Hori and Nemat-Nasser (1994), which have been developed for linearly elastic solids. These results are further generalized to obtain exact expressions for the average field quantities taken over each annulus of a nested sequence of ellipsoidal regions within a finitely (uniformly) deformed infinite solid, where a constant distinct eigenvelocity gradient is prescribed on each annulus; the eigenvelocity gradient within the inner most ellipsoid need not be uniform. 


\subsection{Notation}

For simplicity, a background fixed rectangular Cartesian coordinate system is used. The unit coordinate base vectors are denoted by $\mathbf{e}_{1}, \mathbf{e}_{2}$, and $\mathbf{e}_{3}$. Both subscript and direct notation is used throughout. Depending on the occasion, the unit coordinate triad, $\left(\mathbf{e}_{1}, \mathbf{e}_{2}, \mathbf{e}_{3}\right)$, is collectively denoted by $\mathbf{e}_{i}$ or $\mathbf{e}_{A}, i, A=1,2,3$. Vectors are generally designated by bold-face letters, such as a, b. The scalar product of two vectors is denoted by a dot between them, and the summation convention on repeated indices is used. When a general secondorder tensor $\mathbf{T}$, with components $T_{i j}$, operates on a vector $\mathbf{n}$ with components $n_{i}$, a vector, say, $\mathbf{t}$, with components $t_{i}=T_{i j} n_{j}$, is produced. Throughout, matrix operation rules are used, so that the above operation also has the following direct (coordinateindependent) representation: $\mathbf{t}=\mathbf{T n}$; hence, a second-order tensor followed by a vector implies contraction. The components of $\mathbf{T}$ are $T_{i j}=\mathbf{e}_{i} \cdot \mathbf{T} \mathbf{e}_{j}$. The notation $\mathbf{n} \mathbf{T}$ is also used for $\mathbf{n} \cdot \mathbf{T}$ which is a vector with components $n_{i} T_{i j}$. If $\mathbf{T}=\mathbf{T}(\mathbf{x})$ is a tensor-valued, differentiable function, its divergence is written as, $\boldsymbol{\nabla} \mathbf{T} \equiv \boldsymbol{\nabla} \cdot \mathbf{T}=\mathbf{e}_{i} \partial() / \partial x_{i} \cdot\left(T_{j k} \mathbf{e}_{j} \otimes \mathbf{e}_{k}\right)=$ $\left(\partial T_{i j} / \partial x_{i}\right) \mathbf{e}_{j}$. All vectorial and tensorial quantities are real-valued, i.e., they have real-valued components. The tensorial operation of $\boldsymbol{\nabla}$ on $\mathbf{T}$ is represented by $\boldsymbol{\nabla} \otimes \mathbf{T} \equiv\left(\partial T_{i j} / \partial x_{k}\right) \mathbf{e}_{k} \otimes \mathbf{e}_{i} \otimes \mathbf{e}_{j}$. If $\mathbf{S}$ and $\mathbf{E}$ are two second-order tensors with respective components $S_{i j}$ and $E_{i j}$, then the single and double contraction of their products, respectively are a second-order tensor with components $S_{i j} E_{j k}$ and a scalar $S_{i j} E_{j i}$, i.e., $\mathbf{S E} \equiv \mathbf{S} \cdot \mathbf{E}=S_{i j} E_{j k} \mathbf{e}_{i} \otimes \mathbf{e}_{k}$, and $\operatorname{tr}(\mathbf{S E}) \equiv \mathbf{S}: \mathbf{E}=S_{i j} E_{j i}$. Similarly, if $\mathscr{L}$ is a fourthorder tensor with components $\mathscr{L}_{i j k l}$, then $\mathscr{L} \mathbf{E} \equiv \mathscr{L}: \mathbf{E}=L_{i j l k} E_{k l} \mathbf{e}_{i} \otimes \mathbf{e}_{j}$. The reader should note the order of contractions in these expressions, since alternative conventions are used elsewhere in the literature. ${ }^{2}$ The transpose of a tensor is designated by superscript T. For example, $\mathbf{E}^{\mathrm{T}}$ has components $E_{j i}$ when the components of $\mathbf{E}$ are $E_{i j}$.

\footnotetext{
${ }^{2}$ Nemat-Nasser and Hori (1993), for example, use the convention $\mathscr{L}: \mathbf{E}=\mathscr{L}_{i j k l} E_{k l} \mathbf{e}_{i} \otimes \mathbf{e}_{j}$.
}

\section{Averaging theorems}

There are a number of general averaging theorems, valid at finite deformations and rotations of continua of any arbitrary heterogeneous material composition. These theorems are discussed in this section in terms of the deformation gradient, the nominal stress tensor, and their rates.

Let a deformable body $B$ be in configuration $C_{0}$ at the initial time $t_{0}$. Denote by $C$ its configuration at the current instant $t>t_{0}$. The mapping from $C_{0}$ to $C$ is denoted by $x_{i}=x_{i}\left(X_{A}, t\right)$, or $\mathbf{x}=\mathbf{x}(\mathbf{X}, t)$, which defines particle positions $\mathbf{x}$ of components $x_{i}$ in $C$ in terms of their initial positions $\mathbf{X}$ of components $X_{A}$, both taken with respect to the background rectangular Cartesian coordinate system. Denote the deformation gradient by $\mathbf{F}=$ $(\partial \mathbf{x} / \partial \mathbf{X})^{\mathrm{T}}$.

With $\mathbf{L}=\dot{\mathbf{F}} \mathbf{F}^{-1}=\mathbf{D}+\mathbf{W}$ defining the velocity gradient in reference to the current state, the Kirchhoff stress, $\tau$, the Cauchy stress, $\sigma=\left(\rho / \rho_{0}\right) \tau$, and the nominal stress, $\mathrm{S}^{N}$, are defined such that, Hill (1968),

$$
\frac{1}{\rho_{0}} \operatorname{tr}(\tau \mathbf{D})=\frac{1}{\rho} \operatorname{tr}(\boldsymbol{\sigma} \mathbf{D})=\frac{1}{\rho_{0}} \operatorname{tr}\left(\mathbf{S}^{N} \dot{\mathbf{F}}\right)
$$

represents the rate of stress work per unit mass; here $\rho_{0}$ and $\rho$ are the initial and current mass densities. Hence, the nominal stress, $\mathbf{S}^{N}$, is given by $\mathbf{S}^{N}=\mathbf{F}^{-1} \tau=J \mathbf{F}^{-1} \boldsymbol{\sigma}$.

Other stress tensors, conjugate to other material strain measures, can be defined using the invariance of the rate of stress work per unit mass; Hill (1970). Table 1 summarizes the relation among some commonly used stress measures.

\subsection{Comments on length scales}

The continuum theory of materials deals with objects which on a local level can be regarded as homogeneous, although globally they may be heterogeneous. At a typical material point, an infinitesimal material element is considered, within which the strain and stress are regarded as homogeneous. As a prerequisite, the continuum itself must be locally homogeneous in a certain sense; Hill (1956). 
Table 1

Relations among various stress measures

\begin{tabular}{llllll}
\hline Quantity & $\boldsymbol{\sigma}$ & $\tau$ & $\mathbf{S}^{N}$ & $\mathbf{T}^{\mathrm{R}}$ & $\mathbf{S}^{\mathrm{P}}$ \\
\hline Cauchy $\boldsymbol{\sigma}$ & $\boldsymbol{\sigma}$ & $\frac{1}{J} \tau$ & $\frac{1}{J} \mathbf{F} \mathbf{S}^{N}$ & $\frac{1}{J} \mathbf{T}^{\mathrm{R}} \mathbf{F}^{\mathrm{T}}$ & $\frac{1}{J} \mathbf{F} \mathbf{S}^{\mathrm{P}} \mathbf{F}^{\mathrm{T}}$ \\
Kirchhoff $\tau$ & $J \boldsymbol{\sigma}$ & $\tau$ & $\mathbf{F S}^{N}$ & $\mathbf{T}^{\mathrm{R}} \mathbf{F}^{\mathrm{T}}$ & $\mathbf{F S}^{\mathrm{P}} \mathbf{F}^{\mathrm{T}}$ \\
Nominal $\mathbf{S}^{N}$ & $J \mathbf{F}^{-1} \boldsymbol{\sigma}$ & $\mathbf{F}^{-1} \tau$ & $\mathbf{S}^{N}$ & $\left.\mathbf{T}^{\mathrm{R}}\right)^{\mathrm{T}}$ & $\mathbf{S}^{\mathrm{P}} \mathbf{F}^{\mathrm{T}}$ \\
1 st Piola-Kirchhoff $\mathbf{T}^{\mathrm{R}}$ & $J \boldsymbol{\sigma} \mathbf{F}^{-\mathrm{T}}$ & $\tau \mathbf{F}^{-\mathrm{T}}$ & $\left(\mathbf{S}^{N}\right)^{\mathrm{T}}$ & $\mathbf{T}^{\mathrm{R}}$ & $\mathbf{F S}^{\mathrm{P}}$ \\
2nd Piola-Kirchhoff $\mathbf{S}^{\mathrm{P}}$ & $J \mathbf{F}^{-1} \boldsymbol{\sigma} \mathbf{F}^{-\mathrm{T}}$ & $\mathbf{F}^{-1} \tau \mathbf{F}^{-\mathrm{T}}$ & $\mathbf{S}^{N} \mathbf{F}^{-\mathrm{T}}$ & $\mathbf{F}^{-1} \mathbf{T}^{\mathrm{R}}$ & $\mathbf{S}^{\mathrm{P}}$ \\
\hline
\end{tabular}

In reality, however, materials are heterogeneous, locally, as well as possibly globally. Therefore, whether or not a continuum approach provides useful information depends on the values of two basic relative length scales. These are the greatest dimension of the microconstituents of the aggregate relative to the smallest dimension of the elementary continuum material neighborhood, and the greatest dimension of the elementary continuum material neighborhood relative to the smallest dimension of the overall continuum. For example, in a polycrystalline solid consisting of crystals of tens of microns, each crystal is viewed as a microconstituent. The elementary continuum material neighborhood must then be, at smallest, of the order of fractions of a millimeter, and the overall solid, i.e., the structural component, may have dimensions of the order of centimeters or greater. On the other hand, an earth dam may be viewed as a continuum with aggregates as microconstituents and continuum elements of the order of meters. In either case, the objective is to define an equivalent continuum material element which, in a certain sense, has the same average mechanical response as the actual heterogeneous material element. $^{3}$

To this end, the concept of a representative volume element (RVE) is introduced. In this approach, the actual dimensions of an RVE are of no concern. It is only its dimension relative to the dimension of its essential microstructure that is of importance. If the smallest dimension of the RVE is $D$ and the greatest dimension of its microconstituent is $d$, Hill (1956) suggests that an effective

\footnotetext{
${ }^{3}$ For a more detailed discussion and references, see Hashin (1983) and Nemat-Nasser and Hori (1993).
}

continuum may be produced for $d / D$ less than $10^{-3}$.

\subsection{Choice of deformation and stress measures}

At finite strains and rotations, relations among kinematical and dynamical variables are nonlinear. These relations hold within each grain in an RVE. It is desirable to define the overall average kinematical and dynamical quantities for the RVE such that similar relations remain valid at the macrolevel. The nonlinearity, however, precludes representation of all deformation and stress measures and their rates, as unweighted volume averages over the RVE.

The overall macroscopic quantities, whether obtained by simple unweighted volume averaging or defined in terms of other averaged measures, will be denoted by superposed bars. For example, $\overline{\mathbf{F}}, \overline{\mathbf{L}}, \overline{\mathbf{S}}^{N}$, and $\overline{\boldsymbol{\sigma}}$ are, respectively, the overall measure of the deformation gradient, the velocity gradient, the nominal stress, and the Cauchy stress. When such an overall quantity is an unweighted volume average over the RVE, then it is denoted by $\langle\ldots\rangle$. For example, if the overall deformation gradient is defined by

$\overline{\mathbf{F}}=\frac{1}{V} \int_{V} \mathbf{F}(\mathbf{X}, t) \mathrm{d} V$,

then,

$\overline{\mathbf{F}} \equiv\langle\mathbf{F}\rangle$.

On the other hand, the overall, say, Lagrangian strain is then defined by

$\overline{\mathbf{E}}^{\mathrm{L}} \equiv \frac{1}{2}\left(\overline{\mathbf{F}}^{\mathrm{T}} \overline{\mathbf{F}}-1\right)=\frac{1}{2}\left(\langle\mathbf{F}\rangle^{\mathrm{T}}\langle\mathbf{F}\rangle-1\right)$. 
Clearly, in general, $\overline{\mathbf{E}}^{\mathrm{L}} \neq\left\langle\mathbf{E}^{\mathrm{L}}\right\rangle$, where $\mathbf{E}^{\mathrm{L}} \equiv$ $\left(\mathbf{F}^{\mathrm{T}} \mathbf{F}-1\right) / 2$ is the Lagrangian strain.

In view of the above observation, it is seen that, for finite deformation, there is an inherent arbitrariness in the selection of suitable kinematical and dynamical quantities whose overall measures are defined in terms of unweighted volume averages of the corresponding micromeasures, and which are then employed to define other overall quantities, using the usual continum mechanics relations. Therefore, the selection must be made with care and with due regard for the physical basis of the basic phenomenon and the experimental procedures and results.

In an experimental setting, attempts are made to subject the specimen over its gauge length to measurable uniform deformations and stresses. The uniformity here is in the sense of continuum mechanics. ${ }^{4}$ Thus, it appears natural to consider an RVE subjected to either uniform surface tractions or linear surface displacements, i.e., to uniform boundary data. This will then suggest certain macroscopically uniform deformation and stress measures which are completely described by the boundary data, and which are volume averages of the corresponding nonuniform micromeasures; Hill $(1968,1972)$. It turns out that, for finite deformation, the deformation gradient, $\mathbf{F}$, its rate, $\dot{\mathbf{F}}$, and the nominal stress, $\mathbf{S}^{N}$, and its rate, $\dot{\mathbf{S}}^{N}$, are suitable deformation and stress measures for the purpose of averaging. Their unweighted volume averages are completely defined in terms of the surface data (whether uniform or not), and for either the uniform traction or the linear displacement boundary data, they lead to many useful relations which can be employed to effectively characterize the overall aggregate response. These and related issues are discussed in this section.

\subsection{Average deformation and deformation-rate measures}

Let the deformation of an RVE be defined by the mapping $\mathbf{x}=\mathbf{x}(\mathbf{X}, t)$. The initial reference state

\footnotetext{
${ }^{4}$ At the microlevel, neither the deformation nor the stress is uniform.
}

is any arbitrary configuration, not necessarily the undeformed one. The surface displacements are arbitrary and need not be uniform. The volume average deformation gradient is given in terms of the surface data by

$$
\begin{aligned}
& \overline{\mathbf{F}}=\langle\mathbf{F}\rangle=\frac{1}{V} \int_{\partial V} \mathbf{x} \otimes \mathbf{N} \mathrm{d} A, \\
& \bar{F}_{i B} \equiv\left\langle F_{i B}\right\rangle=\frac{1}{V} \int_{\partial V} x_{i} N_{B} \mathrm{~d} A .
\end{aligned}
$$

This follows from the Gauss theorem. Here, $V$ is the initial volume with surface $\partial V$ of exterior unit normal N. Similarly, for an arbitrary but compatible surface velocity, $\dot{\mathbf{x}}=\dot{\mathbf{x}}(\mathbf{X}, t)$,

$\overline{\dot{\mathbf{F}}} \equiv\langle\dot{\mathbf{F}}\rangle=\frac{1}{V} \int_{\partial V} \dot{\mathbf{x}} \otimes \mathbf{N} \mathrm{d} A \equiv \dot{\overline{\mathbf{F}}}$,

$\bar{F}_{i B} \equiv\left\langle\dot{F}_{i B}\right\rangle=\frac{1}{V} \int_{\partial V} \dot{x}_{i} N_{B} \mathrm{~d} A \equiv \dot{\bar{F}}_{i B}$.

The overall velocity gradient $\overline{\mathbf{L}}$ is then defined by

$\overline{\mathbf{L}} \equiv \dot{\overline{\mathbf{F}}} \overline{\mathbf{F}}^{-1}$.

In general, $\langle\mathbf{L}\rangle \neq \overline{\mathbf{L}}$ when the reference configuration does not coincide with the current one, since $\mathbf{L}=\dot{\mathbf{F}} \mathbf{F}^{-1}$. On the other hand, when the current configuration is chosen as reference, it follows that $\mathbf{F}=\mathbf{1}$, and hence, $\overline{\mathbf{L}}=\langle\mathbf{L}\rangle$. This is an important case, often used for incremental calculation of aggregate properties; Iwakuma and Nemat-Nasser (1984) and Nemat-Nasser and Obata (1986). Note also that, in this case, both the overall average deformation rate and the spin tensors equal the corresponding unweighted volume average, $\overline{\mathbf{D}}=$ $\langle\mathbf{D}\rangle$ and $\overline{\mathbf{W}}=\langle\mathbf{W}\rangle$. In the general case, however, these relations do not hold.

\subsection{Average stress measures}

Let an RVE be subjected to arbitrary but selfequilibrating (zero body forces) surface tractions $\mathbf{T}$, referred to and measured per unit area of some chosen reference configuration. These tractions need not be uniform. Let $\mathbf{S}^{N}=\mathbf{S}^{N}(\mathbf{X}, t)$ be the corresponding variable nominal stress field in the 
RVE, in equilibrium with these boundary tractions. Equilibrium requires, 5

$\boldsymbol{\nabla} \cdot \mathbf{S}^{N}=0$ in $V, \quad \mathbf{N} \cdot \mathbf{S}^{N}=\mathbf{T}$ on $\partial V$,

where $\mathbf{N}$ is the exterior unit normal of $\partial V$. From these conditions and the Gauss theorem, the overall average nominal stress $\overline{\mathbf{S}}^{N}$ is uniquely defined in terms of the boundary tractions by

$\overline{\mathbf{S}}^{N} \equiv\left\langle\mathbf{S}^{N}\right\rangle=\frac{1}{V} \int_{\partial V} \mathbf{X} \otimes \mathbf{T} \mathrm{d} A$.

If the applied self-equilibrating tractions, referred to and measured per unit area of the current configuration, are denoted by $\mathbf{t}$, and if $\boldsymbol{\sigma}=\boldsymbol{\sigma}=(\mathbf{x}, t)$ is the variable Cauchy stress in equilibrium with these tractions, then

$\boldsymbol{\nabla} \cdot \boldsymbol{\sigma}=\mathbf{0}$ in $v$

$\mathbf{n} \cdot \boldsymbol{\sigma}=\mathbf{t}$ on $\partial v$.

Application of the Gauss theorem again shows

$\overline{\boldsymbol{\sigma}} \equiv\langle\boldsymbol{\sigma}\rangle=\frac{1}{v} \int_{\partial v} \mathbf{x} \otimes \mathbf{t} \mathrm{d} a$,

where $v$ is the current volume with boundary $\partial v$. At the local level, the Cauchy and the nominal stresses are related by $\boldsymbol{\sigma}=\mathbf{F S}^{N} / J$. Therefore, if surface data are given with reference to the initial configuration, and the overall nominal stress is defined by Eq. (9), then, in general, $\overline{\mathbf{F}} \overline{\mathbf{S}}^{N} \mid \bar{J} \neq\langle\boldsymbol{\sigma}\rangle$. Hence, once Eq. (9) is chosen for the definition of the overall nominal stress, then Eq. (11) must be abandoned in favor of

$\overline{\boldsymbol{\sigma}} \equiv \frac{1}{\bar{J}} \overline{\mathbf{F}} \overline{\mathbf{S}}^{N}, \quad \bar{J} \equiv \operatorname{det} \overline{\mathbf{F}}$.

On the other hand, when the current configuration is the reference one, all stress measures become identical, and (9) reduces to (11).

\footnotetext{
${ }^{5}$ Even though the stress field may vary in time, in the description of the average properties of an RVE, this variation is viewed as being quasi-static. The inertia forces are then included in the equations of motion of the homogenized RVE.
}

\subsection{Average stress-rate measures}

Let $\dot{\mathbf{T}}$ be the self-equilibrating traction rates (not necessarily uniform) prescribed on $\partial V$, and measured relative to some chosen reference state. Let $\dot{\mathbf{S}}^{N}=\dot{\mathbf{S}}^{N}(\mathbf{X}, t)$ be the corresponding (variable) nominal stress rate in the RVE. Equilibrium requires,

$\boldsymbol{\nabla} \cdot \dot{\mathbf{S}}^{N}=\mathbf{0}$ in $V, \quad \mathbf{N} \cdot \dot{\mathbf{S}}^{N}=\dot{\mathbf{T}}$ on $\partial V$.

From these conditions and the Gauss theorem, the overall average nominal stress rate, $\dot{\mathbf{S}}^{N}$, is uniquely defined in terms of the boundary data by

$\dot{\mathbf{S}}^{N} \equiv\left\langle\dot{\mathbf{S}}^{N}\right\rangle=\frac{1}{V} \int_{\partial V} \mathbf{X} \otimes \dot{\mathbf{T}} \mathrm{d} A \equiv \dot{\mathbf{S}}^{N}$.

This equation is valid whether or not the reference configuration coincides with the current configuration. The overall Cauchy stress rate, $\dot{\bar{\sigma}}$, however, will not enjoy a representation similar to Eq. (14a). That is, while

$\dot{\overline{\mathbf{S}}}^{N} \equiv \frac{\partial}{\partial t}\left\langle\mathbf{S}^{N}\right\rangle=\frac{1}{V} \int_{\partial V} \mathbf{X} \otimes \dot{\mathbf{T}} \mathrm{d} A=\left\langle\dot{\mathbf{S}}^{N}\right\rangle \equiv \overline{\mathbf{S}}^{N}$,

similar relations cannot be written for other stress measures, including the Cauchy stress, even if the current configuration is the reference one. Hence, once Eqs. (9), (14a) and (14b) are accepted as the overall stress and stress rate measures, all other stress and stress rate measures must then be defined in terms of these quantities and the overall deformation gradient $\overline{\mathbf{F}}=\langle\mathbf{F}\rangle$ and its rate $\dot{\dot{\mathbf{F}}} \equiv\langle\dot{\mathbf{F}}\rangle=\dot{\overline{\mathbf{F}}}$, using the usual continuum relations; e.g., expressions given in Table 1 for various stress measures.

\subsection{General identities}

Whatever the material composition, and whatever the deformation and rotation history of an arbitrary heterogeneous material of reference volume $V$, there is a set of fundamental averaging identities that follow from equilibrium, the Gauss theorem, and the definition of the deformation gradient and its rate, for any consistent boundary data; Hill (1984). Consider an arbitrary selfequilibrating nominal stress field, $\mathbf{S}^{N}$, which satis- 
fies the equilibrium conditions Eq. (8), for some self-equilibrating boundary tractions $\mathbf{T}$. Let $\dot{\mathbf{F}}$ in $V$, be the time rate of change of a deformation gradient associated with an arbitrary self-compatible boundary velocity field, $\dot{\mathbf{x}}=\dot{\mathbf{U}}$ on $\partial V$. The chosen reference state can be arbitrary, and the nominal stress, $\mathbf{S}^{N}$, and the deformation rate, $\dot{\mathbf{F}}$, need not be related. ${ }^{6}$ Now, through the application of the Gauss theorem, it follows that ${ }^{7}$

$$
\begin{aligned}
& \left\langle\dot{\mathbf{F}} \mathbf{S}^{N}\right\rangle-\langle\dot{\mathbf{F}}\rangle\left\langle\mathbf{S}^{N}\right\rangle \\
& \quad=\frac{1}{V} \int_{\partial V}(\dot{\mathbf{x}}-\langle\dot{\mathbf{F}}\rangle \mathbf{X}) \otimes\left\{\mathbf{N} \cdot\left(\mathbf{S}^{N}-\left\langle\mathbf{S}^{N}\right\rangle\right)\right\} \mathrm{d} A,
\end{aligned}
$$

where $\dot{\mathbf{x}}=\dot{\mathbf{U}}$ and $\mathbf{N} \cdot \mathbf{S}^{N}=\mathbf{T}$ on $\partial V$.

Similarly, if $\dot{\mathbf{S}}^{N}$ is a self-equilibrating stress rate in $V$, then identity (15a) remains valid when $\mathbf{S}^{N}$ is replaced by $\dot{\mathbf{S}}^{N}$ or if $\dot{\mathbf{F}}$ is replaced by $\mathbf{F}$ (self-compatible) and $\mathbf{S}^{N}$ is replaced by $\dot{\mathbf{S}}^{N}$, or when only $\dot{\mathbf{F}}$ is replaced by $\mathbf{F}$. In this manner, the following identities are obtained:

$$
\begin{aligned}
& \left\langle\mathbf{F} \dot{\mathbf{S}}^{N}\right\rangle-\langle\mathbf{F}\rangle\left\langle\dot{\mathbf{S}}^{N}\right\rangle \\
& \quad=\frac{1}{V} \int_{\partial V}(\mathbf{x}-\langle\mathbf{F}\rangle \mathbf{X}) \otimes\left\{\mathbf{N} \cdot\left(\dot{\mathbf{S}}^{N}-\left\langle\dot{\mathbf{S}}^{N}\right\rangle\right)\right\} \mathrm{d} A, \\
& \left\langle\mathbf{F S}^{N}\right\rangle-\langle\mathbf{F}\rangle\left\langle\mathbf{S}^{N}\right\rangle \\
& \quad=\frac{1}{V} \int_{\partial V}(\mathbf{x}-\langle\mathbf{F}\rangle \mathbf{X}) \otimes\left\{\mathbf{N} \cdot\left(\dot{\mathbf{S}}^{N}-\left\langle\mathbf{S}^{N}\right\rangle\right)\right\} \mathrm{d} A, \\
& \left\langle\dot{\mathbf{F}} \dot{\mathbf{S}}^{N}\right\rangle-\langle\dot{\mathbf{F}}\rangle\left\langle\dot{\mathbf{S}}^{N}\right\rangle \\
& \quad=\frac{1}{V} \int_{\partial V}(\dot{\mathbf{x}}-\langle\dot{\mathbf{F}}\rangle \mathbf{X}) \otimes\left\{\mathbf{N} \cdot\left(\dot{\mathbf{S}}^{N}-\left\langle\dot{\mathbf{S}}^{N}\right\rangle\right)\right\} \mathrm{d} A .
\end{aligned}
$$

\footnotetext{
${ }^{6}$ Similar comments apply to $\mathbf{F}$ and $\dot{\mathbf{S}}^{N}$ in Eqs. (15b)-(15d).

${ }^{7}$ The trace of the first term in Eq. (15a) is the rate of stress work per unit reference volume, when the stress and deformation rate correspond to each other.
}

In the same manner, it is shown that,

$$
\begin{aligned}
& \left\langle\dot{\mathbf{F}} \mathbf{S}^{N}\right\rangle-\langle\dot{\mathbf{F}}\rangle\left\langle\mathbf{S}^{N}\right\rangle \\
& \quad=\frac{1}{V} \int_{\partial V}(\dot{\mathbf{x}}-\langle\dot{\mathbf{F}}\rangle \mathbf{X}) \otimes\left(\mathbf{N} \cdot \mathbf{S}^{N}\right) \mathrm{d} A \\
& \left\langle\mathbf{F} \dot{\mathbf{S}}^{N}\right\rangle-\langle\mathbf{F}\rangle\left\langle\dot{\mathbf{S}}^{N}\right\rangle \\
& \quad=\frac{1}{V} \int_{\partial V}(\mathbf{x}-\langle\mathbf{F}\rangle \mathbf{X}) \otimes\left(\mathbf{N} \cdot \dot{\mathbf{S}}^{N}\right) \mathrm{d} A \\
& \left\langle\mathbf{F S}^{N}\right\rangle-\langle\mathbf{F}\rangle\left\langle\mathbf{S}^{N}\right\rangle \\
& \quad=\frac{1}{V} \int_{\partial V}(\mathbf{x}-\langle\mathbf{F}\rangle \mathbf{X}) \otimes\left(\mathbf{N} \cdot \mathbf{S}^{N}\right) \mathrm{d} A \\
& \left\langle\dot{\mathbf{F}} \dot{\mathbf{S}}^{N}\right\rangle-\langle\dot{\mathbf{F}}\rangle\left\langle\dot{\mathbf{S}}^{N}\right\rangle \\
& \quad=\frac{1}{V} \int_{\partial V}(\dot{\mathbf{x}}-\langle\dot{\mathbf{F}}\rangle \mathbf{X}) \otimes\left(\mathbf{N} \cdot \dot{\mathbf{S}}^{N}\right) \mathrm{d} A
\end{aligned}
$$

and that,

$$
\begin{aligned}
& \left\langle\dot{\mathbf{F}} \mathbf{S}^{N}\right\rangle-\langle\dot{\mathbf{F}}\rangle\left\langle\mathbf{S}^{N}\right\rangle \\
& =\frac{1}{V} \int_{\partial V} \mathbf{x} \otimes\left\{\mathbf{N} \cdot\left(\mathbf{S}^{N}-\left\langle\mathbf{S}^{N}\right\rangle\right)\right\} \mathrm{d} A, \\
& \left\langle\mathbf{F} \dot{\mathbf{S}}^{N}\right\rangle-\langle\dot{\mathbf{F}}\rangle\left\langle\dot{\mathbf{S}}^{N}\right\rangle \\
& \quad=\frac{1}{V} \int_{\partial V} \mathbf{x} \otimes\left\{\mathbf{N} \cdot\left(\dot{\mathbf{S}}^{N}-\left\langle\dot{\mathbf{S}}^{N}\right\rangle\right)\right\} \mathrm{d} A, \\
& \left\langle\mathbf{F} \mathbf{S}^{N}\right\rangle-\langle\mathbf{F}\rangle\left\langle\mathbf{S}^{N}\right\rangle \\
& \quad=\frac{1}{V} \int_{\partial V} \mathbf{x} \otimes\left\{\mathbf{N} \cdot\left(\mathbf{S}^{N}-\left\langle\mathbf{S}^{N}\right\rangle\right)\right\} \mathrm{d} A, \\
& \left\langle\dot{\mathbf{F}} \dot{\mathbf{S}}^{N}\right\rangle-\langle\dot{\mathbf{F}}\rangle\left\langle\dot{\mathbf{S}}^{N}\right\rangle \\
& \quad=\frac{1}{V} \int_{\partial V} \dot{\mathbf{x}} \otimes\left\{\mathbf{N} \cdot\left(\left\{\dot{\mathbf{S}}^{N}-\left\langle\dot{\mathbf{S}}^{N}\right\rangle\right)\right\} \mathrm{d} A .\right.
\end{aligned}
$$

When the boundary data are such that the integrals on the right-hand side of, say, (15) vanish, then it follows that the average of the corresponding product equals the product of the averages. ${ }^{8}$ This leads to the following useful results:

\footnotetext{
${ }^{8}$ For linearly elastic composites, the corresponding expressions are known as the Hill conditions. A similar association is appropriate for the present expressions.
} 


$$
\begin{aligned}
\left\langle\dot{\mathbf{F}} \mathbf{S}^{N}\right\rangle & =\langle\dot{\mathbf{F}}\rangle\left\langle\mathbf{S}^{N}\right\rangle, \\
\left\langle\mathbf{F} \dot{\mathbf{S}}^{N}\right\rangle & =\langle\mathbf{F}\rangle\left\langle\dot{\mathbf{S}}^{N}\right\rangle, \\
\left\langle\mathbf{F} \mathbf{S}^{N}\right\rangle & =\langle\mathbf{F}\rangle\left\langle\mathbf{S}^{N}\right\rangle, \\
\left\langle\dot{\mathbf{F}} \dot{\mathbf{S}}^{N}\right\rangle & =\langle\dot{\mathbf{F}}\rangle\left\langle\dot{\mathbf{S}}^{N}\right\rangle .
\end{aligned}
$$

\subsection{Uniform boundary tractions and traction rates}

Suppose now the prescribed surface tractions are uniform, so that

$\mathbf{T}=\mathbf{N} \cdot \mathbf{S}^{N 0}$ or $T_{i}=S_{A i}^{N 0} N_{A}$ on $\partial V$,

$\dot{\mathbf{T}}=\mathbf{N} \cdot \dot{\mathbf{S}}^{N 0} \quad$ or $\quad \dot{T}_{i}=\dot{S}_{A i}^{N 0} N_{A}$ on $\partial V$,

where $\mathbf{S}^{N 0}$ and $\dot{\mathbf{S}}^{N 0}$ are spatially uniform, possibly time-dependent (parametrically) tensors. It then follows from Eqs. (9), (14a) and (14b) that,

$\overline{\mathbf{S}}^{N}=\mathbf{S}^{N 0} \quad$ and $\quad \overline{\mathbf{S}}^{N}=\dot{\overline{\mathbf{S}}}^{N}=\dot{\mathbf{S}}^{N 0}$.

The Kirchhoff (see Table 1) stress, $\tau$, relates to the nominal stress tensor, $\mathbf{S}^{N}$, by $\tau=\mathbf{F S}^{N}$, where $\mathbf{F}$ is the corresponding deformation gradient. As pointed out before, in general, the overall average Kirchhoff stress must be defined in terms of the average deformation gradient, $\overline{\mathbf{F}}$, and nominal stress, $\overline{\mathbf{S}}^{\mathrm{N}}$, because, in general, $\bar{\tau}=\left\langle\mathbf{F S}^{N}\right\rangle$ $\neq\langle\mathbf{F}\rangle\left\langle\mathbf{S}^{\mathrm{N}}\right\rangle$. For uniform boundary tractions and traction rates, on the other hand, it is seen that the overall average Kirchhoff stress and its rate enjoy the usual relations,

$$
\begin{aligned}
& \bar{\tau} \equiv\langle\tau\rangle=\left\langle\mathbf{F} \mathbf{S}^{N}\right\rangle=\overline{\mathbf{F}} \mathbf{S}^{N 0}, \\
& \bar{\tau} \equiv\langle\dot{\boldsymbol{\tau}}\rangle=\left\langle\dot{\mathbf{F}} \mathbf{S}^{N}+\mathbf{F} \dot{\mathbf{S}}^{N}\right\rangle=\dot{\overline{\mathbf{F}}} \mathbf{S}^{N 0}+\overline{\mathbf{F}} \dot{\mathbf{S}}^{N 0} \equiv \dot{\bar{\tau}} .
\end{aligned}
$$

These relations follow from Eq. (18).

\subsection{Linear boundary displacements and displace- ment rates}

Suppose an RVE is subjected to linear boundary displacements and velocities, such that

$\mathbf{x}=\mathbf{F}^{0} \mathbf{X} \quad$ and $\quad \dot{\mathbf{x}}=\dot{\mathbf{F}}^{0} \mathbf{X}$ on $\partial V$,

where $\mathbf{F}^{0}$ and $\dot{\mathbf{F}}^{0}$ are possibly time-dependent, but spatially uniform deformation and velocity gradients, measured with respect to some initial reference configuration. Direct substitution into Eqs. (5) and (6) reveals,
$\overline{\mathbf{F}}=\mathbf{F}^{0} \quad$ and $\quad \dot{\overline{\mathbf{F}}}=\dot{\mathbf{F}}^{0}$.

Because of the microheterogeneities of the RVE, $\mathbf{F}$ and $\dot{\mathbf{F}}$ are both, in general, spatially nonuniform, $\mathbf{F}=\mathbf{F}(\mathbf{X}, t)$ and $\dot{\mathbf{F}}=\dot{\mathbf{F}}(\mathbf{X}, t)$. In this case, again, it is easy to show that the corresponding overall average Kirchhoff stress and its rate, satisfy the relations,

$\bar{\tau}=\mathbf{F}^{0} \overline{\mathbf{S}}^{N}, \quad \overline{\dot{\tau}}=\dot{\mathbf{F}}^{0} \overline{\mathbf{S}}^{N}+\mathbf{F}^{0} \overline{\mathbf{S}}^{N}$.

These follow from Eq. (18).

\subsection{General identities for uniform boundary data}

When the boundary data are uniform, then the integrals in the right-hand side of Eqs. (15a)-(15d) to Eq. (17) vanish identically. This leads to identities Eq. (18), with the right-hand side appropriately specialized. Hence, for uniform boundary tractions and traction rates, it follows that,

$$
\begin{aligned}
\left\langle\dot{\mathbf{F}} \mathbf{S}^{N}\right\rangle=\langle\dot{\mathbf{F}}\rangle \mathbf{S}^{N 0}, & \left\langle\mathbf{F} \dot{\mathbf{S}}^{N}\right\rangle=\langle\mathbf{F}\rangle \dot{\mathbf{S}}^{N 0}, \\
\left\langle\mathbf{F} \mathbf{S}^{N}\right\rangle=\langle\mathbf{F}\rangle \mathbf{S}^{N 0}, & \left\langle\dot{\mathbf{F}} \dot{\mathbf{S}}^{N}\right\rangle=\langle\dot{\mathbf{F}}\rangle \dot{\mathbf{S}}^{N 0} .
\end{aligned}
$$

Similarly, for uniform surface displacements and their rates, identities Eq. (18) reduce to

$$
\begin{array}{rlrl}
\left\langle\dot{\mathbf{F}} \mathbf{S}^{N}\right\rangle & =\dot{\mathbf{F}}^{0}\left\langle\mathbf{S}^{N}\right\rangle, & \left\langle\mathbf{F} \dot{\mathbf{S}}^{N}\right\rangle=\mathbf{F}^{0}\left\langle\dot{\mathbf{S}}^{N}\right\rangle, \\
\left\langle\mathbf{F} \mathbf{S}^{N}\right\rangle=\mathbf{F}^{0}\left\langle\mathbf{S}^{N}\right\rangle, & \left\langle\dot{\mathbf{F}} \dot{\mathbf{S}}^{N}\right\rangle=\dot{\mathbf{F}}^{0}\left\langle\dot{\mathbf{S}}^{N}\right\rangle .
\end{array}
$$

\section{Homogenization and concentration tensors}

It is common to assume that the stress and deformation fields are uniform within each grain of a suitably large aggregate representing an RVE, since, by necessity, the grains must be very small relative to the size of the RVE. Such an assumption is in accord with the usual continuum formulation of the flow and deformation of matter. It is also the basis of the general theory of crystals and polycrystals; Rice (1971), Hill and Rice (1972), Hill and Havner (1982), Iwakuma and NematNasser (1984), and Havner (1992).

Consider an RVE subjected to suitable uniform boundary data. The stress and deformation measures are, in general, nonuniform within the RVE. Thus, even though these measures are assumed to 
be uniform over each grain, they change from grain to grain. A fundamental issue is to calculate the local quantities, i.e., the quantities within a typical grain, in terms of suitable uniform data prescribed on the boundary of the RVE. This issue is addressed in this section.

\subsection{Eshelby's tensor}

For infinitesimal deformations, Eshelby (1957) tensor is often used to estimate the local quantities in terms of their overall values. Eshelby considered an ellipsoidal inclusion within an infinitely extended linearly elastic homogeneous solid. He then showed that, if the inclusion undergoes a transformation resulting in a uniform transformation strain, $\boldsymbol{\varepsilon}^{*}$, when free from the constraints imposed by the surrounding matrix, then, in the presence of such a constraint, the strain in the transformed inclusion is still uniform and is given by ${ }^{9} \boldsymbol{\varepsilon}=\mathscr{S}: \boldsymbol{\varepsilon}^{*}$ or, in component form, by $\varepsilon_{i j}=\mathscr{S}_{i j k l} \varepsilon_{l k}^{*}$. Here $\boldsymbol{\varepsilon}$ is the linearized strain, i.e., the symmetric part of the displacement gradient, and $\mathscr{S}$ is Eshelby's tensor, symmetric only with respect to the exchange of $i$ and $j$, and $k$ and $l$, but not in general, of $i j$ and $k l$. This tensor depends on the elastic properties of the matrix and the aspect ratios of the ellipsoid, but not on the properties of the ellipsoid nor the origin of the transformation strain. It has been calculated for isotropic and certain anisotropic cases, and has been used extensively to estimate aggregate properties and to solve various problems in infinitesimal deformation theories; Kröner (1958, 1961), Budiansky and Wu (1962), Hutchinson (1970), and others; see, e.g., Mura (1987) and NematNasser and Hori (1993) for discussion and other references. Again, for infinitesimal deformations, the idea has been used to obtain average properties of composites with periodic microstructures, for linearly elastic, power-law creep, and elasto-plastic solids; see Nemat-Nasser et al. (1982), and Iwakuma and Nemat-Nasser (1983). A comprehensive

\footnotetext{
${ }^{9}$ This fact appears to have been discovered by Hardiman (1954) in connection with elliptical inclusions in an infinite elastic plates subjected to uniform farfield inplane tractions.
}

account is given by Nemat-Nasser and Hori (1993).

In general, an estimate of the overall properties of an aggregate at finite deformations demands an incremental formulation. It is expedient and theoretically precise, to cast this formulation in terms of the rate of change of the field quantities measured in terms of the actual time for rate-dependent deformations, or some other monotonically changing parameter, when appropriate (e.g., for rate-independent models of plasticity). As pointed out before, large deformation elastoplasticity problems are most effectively formulated in terms of the rate of change of the nominal stress and the velocity gradient, using the current configuration as reference. In this context, Eshelby's technique has been exploited by Iwakuma and Nemat-Nasser (1984) and Nemat-Nasser and Obata (1986) for finite strains and rotations, to construct an explicit procedure for estimating the local rate quantities in terms of their overall average values. In particular, Iwakuma and Nemat-Nasser show that, for an ellipsoidal grain, $\Omega$, embedded in a uniform matrix (both fully nonlinear, having undergone finite deformations), the velocity gradient, $L_{i j}$, is uniform and is given by $L_{i j}=\mathscr{A}_{i j k l}^{\Omega} L_{l k}^{0}$, where $\mathbf{L}^{0}$ is the uniform velocity gradient prescribed at infinity, and $\mathscr{A}^{\Omega}$ is the concentration tensor, for whose calculation explicit formulas are given by Iwakuma and Nemat-Nasser (1984). The technique applies to both rate-independent and rate-dependent elasto-plastic deformations at finite strains. In this approach, the average nominal stress rate and the average velocity gradient are obtained using the current aggregate configuration as the reference one, leading to an estimate of the instantaneous overall pseudo-moduli.

An alternative approach is to perform the averaging, using the initial aggregate configuration for reference. Hence, the nominal stress and its rate, $\mathbf{S}^{\mathrm{N}}$ and $\dot{\mathbf{S}}^{\mathrm{N}}$, as well as the deformation gradient and its rate, $\mathbf{F}$ and $\dot{\mathbf{F}}$, are measured with respect to the initial configuration of the aggregate. When the grains are modeled by ellipsoids embedded in a homogenized matrix in order to calculate the concentration tensor, $\mathscr{A}^{\Omega}$, and if all the grains initially have the same aspect ratio and the same orientation, then a certain simplification 
is attained in the averaging process. ${ }^{10}$ This advantage is lost if the initial configuration of the grains does not lend itself to such simplification. Furthermore, in finite-deformation elastoplasticity, the overall incremental displacement gradients (velocity gradients) or the boundary traction increments (traction rates) are generally prescribed in reference to the current deformed state. Similarly, the deformation rate and the stress rate of each grain are naturally and conveniently measured with respect to the current configuration. As pointed out before, when the current configuration is used as the reference one, then the overall velocity gradient is given by the simple volume average of the variable velocity gradient of the aggregates, because, in this case, the deformation gradient, relative to the current configuration, is the identity tensor; i.e., when the current configuration, say, at the time $t$, is used as the reference configuration, then

$\overline{\mathbf{L}}=\langle\mathbf{L}\rangle=\left\langle\dot{\mathbf{F F}}_{t}^{-1}\right\rangle=\left\langle\dot{\mathbf{F}}_{t}\right\rangle, \quad \mathbf{F}_{t}=\mathbf{1}$,

where the subscript $t$ emphasizes that the configuration at current time $t$ is the reference one. Hence, if the boundary data for the RVE are given by

$\dot{\boldsymbol{x}}=\dot{\mathbf{F}}_{t}^{0} \mathbf{x}$ on $\partial v$,

using the current configuration with boundary $\partial v$ as reference, then it immediately follows that

$\overline{\mathbf{L}}=\dot{\mathbf{F}}_{t}^{0}$,

$\overline{\mathbf{F}}_{t}=\dot{\mathbf{F}}_{t}^{0}$.

This attractive exact kinematical relation is lost when a fixed initial configuration is used for referencing purposes. Indeed, if the boundary data at an instant $t$ are given by

$\dot{\mathbf{x}}=\dot{\mathbf{F}}^{0} \mathbf{X}$ on $\partial V$,

with $\dot{\mathbf{F}}^{0}$ being a prescribed constant tensor, and $\partial V$ referring to the boundary of the initial configuration of the aggregate, it then follows that

\footnotetext{
${ }^{10}$ This has been pointed out by Harren (1991) who uses initially spherical grains for the purpose of averaging.
}

$\overline{\mathbf{\mathbf { F }}}=\dot{\mathbf{F}}^{0}$,

whereas

$\overline{\mathbf{L}} \equiv\langle\mathbf{L}\rangle=\left\langle\dot{\mathbf{F}} \mathbf{F}^{-1}\right\rangle \neq \dot{\mathbf{F}}^{0} \overline{\mathbf{F}}^{-1}$.

In this case, therefore, one must define $\overline{\mathbf{L}}$ by $\dot{\mathbf{F}}^{0} \overline{\mathbf{F}}^{-1}$, which introduces certain inconsistencies into the self-consistent averaging process. Note, however, that Eq. (27) and Eqs. (28a)-(28c) may be viewed to be special cases of Eqs. (29a)-(29c), obtained from the latter by identifying the reference state with the current configuration. In the following sections, therefore, an arbitrary reference configuration is used to obtain general results, and, then, these are specialized, wherever necessary.

\subsection{General phase-transformation problem}

Consider an arbitrary reference configuration. The nominal stress rate and the velocity gradient, denoted by $\dot{\mathbf{S}}^{N}$ and $\dot{\mathbf{F}}$, with components $\dot{S}_{A i}^{N}$ and $\dot{F}_{i A}$, are used as the basic stress rate and deformation rate measures. These quantities are assumed to be connected through certain instantaneous pseudo-moduli. More specifically, consider an extended homogeneous solid of reference volume $V$, with instantaneous modulus tensor $\mathscr{F}$, of components $\mathscr{F}_{A i B j}$. Assume that the nominal stress rate, $\dot{\mathbf{S}}^{\mathrm{N}}$, is a linear and homogeneous function of the velocity gradient, $\dot{\mathbf{F}}$, both being referred to the same arbitrary reference configuration, i.e., set ${ }^{11}$

$\dot{\mathbf{S}}^{N}=\mathscr{F}: \dot{\mathbf{F}} \quad$ or $\quad \dot{S}_{A i}^{N}=\mathscr{F}_{A i B j} \dot{F}_{j B}$,

where $i, j, A, B=1,2,3$, and the summation convention is used. In many applications, the pseudo-modulus tensor $\mathscr{F}$ is symmetric with respect to the exchange of $A i$ and $B j$, but not with respect to the exchange of $A$ and $i$ or $B$ and $j$. The former symmetry property will be used in the sequel wherever appropriate.

\footnotetext{
${ }^{11}$ Since neither $\dot{F}_{i A}$ nor $\dot{S}_{A i}^{N}$ is symmetric, care must be taken in using coordinate-invariant contractions. Here, the double contraction denoted by:, is defined in Eqs. (30a)-(30c) and will be followed throughout this work.
} 
Suppose that the pseudo-modulus $\mathscr{F}$ admits an inverse, $\mathscr{G}$, so that

$\mathscr{F}: \mathscr{G}=\mathbf{1}^{(4)}, \quad$ or $\quad \mathscr{F}_{A i B j} \mathscr{G}_{j B k C}=\delta_{A C} \delta_{i k}$,

where $\mathbf{1}^{(4)}$ with components $\delta_{i j} \delta_{A B}$, is the general, fourth-order identity tensor. Then, it follows that

$\dot{\mathbf{F}}=\mathscr{G}: \dot{\mathbf{S}}^{N}, \quad \dot{F}_{i A}=\mathscr{G}_{i A j B} \dot{S}_{B j}^{N}$.

The tensor $\mathscr{G}$ with components $\mathscr{G}_{i A j B}$ will be referred to as the pseudo-compliance. Its symmetry properties are the same as those of $\mathscr{F}$.

Let now a region $\Omega$ in $V$ undergo a phase transformation which, if $\Omega$ were free from the constraint imposed by the surrounding material, ${ }^{12}$ it would attain a constant transformation (inelastic) velocity gradient $\dot{\mathbf{F}}^{*}$ with components $\dot{F}_{i A}^{*}$. Let the resulting velocity gradient of $\Omega$ in the presence of the constraint from the surrounding matrix, be $\dot{\mathbf{F}}$ with components $\dot{F}_{i A}$. In general, $\dot{\mathbf{F}}$ is spatially nonuniform. However, when $V$ is homogeneous and unbounded, and $\Omega$ is ellipsoidal, then, following Eshelby's procedure, it is shown by Iwakuma and Nemat-Nasser (1984) that the resulting final velocity gradient $\dot{\mathbf{F}}$ in $\Omega$ (for any constant transformation velocity gradient $\dot{\mathbf{F}}^{*}$ ) is constant; see Section 4.5 . In this case, $\dot{\mathbf{F}}$ is related linearly to the transformation velocity gradient $\dot{\mathbf{F}}^{*}$ by

$\dot{\mathbf{F}}=\mathscr{S}: \dot{\mathbf{F}}^{*} \quad$ or $\quad \dot{F}_{i A}=\mathscr{S}_{i A B j} \dot{F}_{j B}^{*}$.

The fourth-order tensor $\mathscr{S}$, in general, has no symmetries. It generalizes Eshelby's tensor which is mentioned in Section 3.1.

Iwakuma and Nemat-Nasser (1984) outline a method for computing $\mathscr{S}$ for a general ellipsoidal $\Omega$. When the operator $\mathscr{F}_{A i B j} \partial^{2}(\ldots) /\left(\partial X_{A} \partial X_{B}\right)$ is elliptic, a real-valued tensor $\mathscr{S}$ exists and can be computed in terms of the aspect ratios of the ellipsoid $\Omega$ and the pseudo-modulus tensor $\mathscr{F}_{A i B j}$. For the sake of referencing, in what follows, the tensor $\mathscr{S}$ with components $\mathscr{S}_{i A B j}$ will be called the generalized Eshelby tensor.

Since $\dot{\mathbf{F}}^{*}$ is the stress-free inelastic velocity gradient in $\Omega$, the nominal stress rate in this region is

\footnotetext{
${ }^{12}$ I.e., if $\Omega$ is cut out and allowed to change without any constraints imposed on its boundary $\partial \Omega$, then its velocity gradient would be $\dot{\mathbf{F}}^{*}$.
}

produced by the differential velocity gradient $\left(\dot{\mathbf{F}}-\dot{\mathbf{F}}^{*}\right)$, and it follows that

$\dot{\mathbf{S}}^{N}=\mathscr{\mathscr { F }}:\left(\dot{\mathbf{F}}-\dot{\mathbf{F}}^{*}\right)=\mathscr{\mathscr { F }}:\left(\mathscr{S}-\mathbf{1}^{(4)}\right): \mathbf{F}^{*}$ in $\Omega$

or, in component form,

$$
\begin{aligned}
\dot{S}_{A i}^{N} & =\mathscr{F}_{A i B j}\left(\dot{F}_{j B}-\dot{F}_{j B}^{*}\right) \\
& =\mathscr{F}_{A i B j}\left(\mathscr{S}_{j B C k}-\delta_{B C} \delta_{j k}\right) \dot{F}_{k C}^{*} \text { in } \Omega .
\end{aligned}
$$

It is convenient to define a transformation nominal stress rate by

$\dot{\mathbf{S}}^{N *} \equiv-\mathscr{F}: \dot{\mathbf{F}}^{*} \quad$ or $\quad \dot{S}_{A i}^{N *} \equiv-\mathscr{F}_{A i B j} \dot{F}_{j B}^{*}$.

Then, Eq. (32a) becomes

$\dot{\mathbf{S}}^{N}=\mathscr{F}: \dot{\mathbf{F}}+\dot{\mathbf{S}}^{N *}$.

\subsection{Homogenization and eigenvelocity gradient}

The transformation velocity gradient, $\dot{\mathbf{F}}^{*}$, and the generalized Eshelby tensor, $\mathscr{S}$, can be used to homogenize an unbounded $V$ of pseudo-modulus tensor $\mathscr{F}$, which contains an ellipsoidal inhomogeneity $\Omega$ of pseudo-modulus tensor $\mathscr{P}^{\Omega}$, and which is subjected to farfield uniform nominal stress rate $\dot{\mathbf{S}}^{N 0}$. Denote the corresponding farfield uniform velocity gradient by $\dot{\mathbf{F}}^{0}$, and note that

$\dot{\mathbf{S}}^{N 0}=\mathscr{F}: \dot{\mathbf{F}}^{0} \quad$ or $\quad \dot{\mathbf{S}}_{A i}^{N 0}=\mathscr{F}_{A i B j} \dot{F}_{j B}^{0}$.

If the solid were uniform throughout its entire volume, then the nominal stress rate field and hence the corresponding velocity gradient field would be uniform when the farfield data are uniform. These fields would be given by $\dot{\mathbf{F}}^{0}$ and $\dot{\mathbf{S}}^{N 0} \equiv \mathscr{\mathscr { F }}: \dot{\mathbf{F}}^{0}$, respectively. The presence of region $\Omega$ with a different pseudo-modulus tensor, i.e., the existence of a material mismatch, disturbs the uniform nominal stress rate and the velocity gradient fields. Denote the resulting variable velocity gradient and nominal stress rate fields, respectively, by $\dot{\mathbf{F}}(\mathbf{X})$ and $\dot{\mathbf{S}}^{\mathrm{N}}(\mathbf{X})$, and set

$\dot{\mathbf{F}}(\mathbf{X})=\dot{\mathbf{F}}^{0}+\dot{\mathbf{F}}^{d}(\mathbf{X})$,

$\dot{\mathbf{S}}^{N}(\mathbf{X})=\dot{\mathbf{S}}^{N 0}+\dot{\mathbf{S}}^{N d}(\mathbf{X})$.

Here, $\dot{\mathbf{F}}^{d}(\mathbf{X})$ and $\dot{\mathbf{S}}^{N d}(\mathbf{X})$ are the disturbance velocity gradient and the nominal stress rate fields caused by the presence of the inclusion $\Omega$, with 


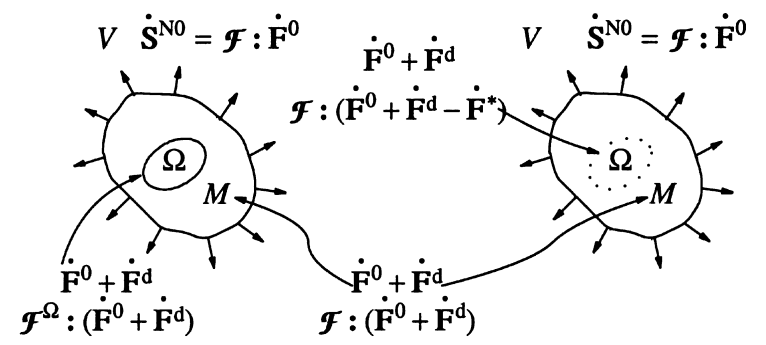

(a) Original

(b) Homogenized

Fig. 1. Equivalent homogeneous solid and eigenvelocity gradient.

mismatched pseudo-moduli. The fields $\dot{\mathbf{S}}^{N}(\mathbf{X})$ and $\dot{\mathbf{F}}(\mathbf{X})$ are related through (Fig. 1(a))

$\dot{\mathbf{S}}^{N}(\mathbf{X})= \begin{cases}\mathscr{F}^{\Omega}: \dot{\mathbf{F}} & \text { in } \Omega \\ \mathscr{F}: \mathbf{F} & \text { in } V-\Omega\end{cases}$

or, in component form,

$\dot{\mathbf{S}}_{A i}^{N}(\mathbf{X})= \begin{cases}\mathscr{F}_{A i B j}^{\Omega} \dot{F}_{j B}(\mathbf{X}) & \text { in } \Omega \\ \mathscr{F}_{A i B j} \dot{F}_{j B}(\mathbf{X}) & \text { in } V-\Omega .\end{cases}$

Equilibrium requires

$\dot{S}_{A i . A}^{N}=0$ in $V$,

$\dot{\mathbf{S}}^{N}(\mathbf{X}) \rightarrow \dot{\mathbf{S}}^{N 0}$ as $|\mathbf{X}| \rightarrow \infty$.

From Eqs. (37a, b) and Eqs. (30a)-(30c), it also follows that

$\dot{\mathbf{F}}(\mathbf{X}) \rightarrow \dot{\mathbf{F}}^{0}$ as $|\mathbf{X}| \rightarrow \infty$.

To solve this boundary-value problem, Eq. (37a) must be integrated in conjunction with Eq. (36a) subject to the farfield conditions (37b) or $(37 \mathrm{c})$ and the continuity of the traction rates and the velocity field across the boundary $\partial \Omega$. Instead of dealing with this rather complex problem, it is convenient and effective to consider an equivalent homogeneous solid which has the uniform pseudomodulus tensor $\mathscr{F}$ of the matrix material everywhere, including in $\Omega$. Then, in order to account for the mismatch of the material properties of the inclusion and the matrix, a suitable velocity gradient field, ${ }^{13} \dot{\mathbf{F}}^{*}(\mathbf{X})$, is introduced in $\Omega$, such that the

\footnotetext{
${ }^{13}$ I.e., inelastic transformation velocity gradient.
}

equivalent homogeneous solid has the same velocity gradient and nominal stress rate fields as the actual heterogeneous solid under the prescribed farfield data. The velocity gradient field, $\dot{\mathbf{F}}^{*}$, necessary for this homogenization will be called the eigenvelocity gradient.

Fig. 1(b) illustrates this procedure for the case when the boundary traction rates, corresponding to the constant farfield nominal stress rate $\mathbf{S}^{N 0}$, are prescribed on $\partial V$. In this figure the eigenvelocity gradient field is given by

$\dot{\mathbf{F}}^{*}(\mathbf{X})= \begin{cases}\mathbf{0} & \text { in } M \\ \dot{\mathbf{F}}^{*} & \text { in } \Omega,\end{cases}$

where $M=V-\Omega$. For this equivalent problem, the pseudo-modulus tensor is uniform everywhere, including in $\Omega$. It is given by $\mathscr{T}$. Therefore, the corresponding nominal stress rate field becomes, in view of Eqs. (35a) and (35b),

$$
\begin{aligned}
\dot{\mathbf{S}}^{N}(\mathbf{X}) & =\mathscr{\mathscr { F }}:\left(\dot{\mathbf{F}}(\mathbf{X})-\dot{\mathbf{F}}^{*}(\mathbf{X})\right) \\
& = \begin{cases}\mathscr{\mathscr { F }}:\left(\dot{\mathbf{F}}^{0}+\dot{\mathbf{F}}^{d}(\mathbf{X})\right) & \text { in } M \\
\mathscr{F}:\left(\dot{\mathbf{F}}^{0}+\dot{\mathbf{F}}^{d}(\mathbf{X})-\dot{\mathbf{F}}^{*}(\mathbf{X})\right) & \text { in } \Omega .\end{cases}
\end{aligned}
$$

Noting Eqs. (35a) and (38a), observe from Eq. (38b) that the disturbance fields are related through

$\dot{\mathbf{S}}^{N d}(\mathbf{X})=\mathscr{\mathscr { F }}:\left(\dot{\mathbf{F}}^{d}(\mathbf{X})-\dot{\mathbf{F}}^{*}(\mathbf{X})\right)$ in $\Omega$.

Furthermore, in the homogenized solid, the velocity gradient $\dot{\mathbf{F}}^{d}(\mathbf{X})$ is produced by the eigenvelocity gradient $\dot{\mathbf{F}}^{*}$. From Eq. (31) it now follows that, for an ellipsoidal $\Omega$,

$\dot{\mathbf{F}}^{d}=\mathscr{S}: \dot{\mathbf{F}}^{*} \quad$ or $\quad \dot{F}_{i A}^{d}=\mathscr{S}_{i A B j} \dot{F}_{j B}^{*}$ in $\Omega$.

The resulting nominal stress rate in $\Omega$ then is

$\dot{\mathbf{S}}^{N d}=\mathscr{\mathscr { F }}:\left(\mathscr{S}-\mathbf{1}^{(4)}\right): \dot{\mathbf{F}}^{*}$

or, in component form,

$\dot{\mathbf{S}}_{A i}^{N d}=\mathscr{F}_{A i B j}\left(\mathscr{S}_{j B C k}-\delta_{B C} \delta_{j k}\right) \dot{F}_{k C}^{*}$.

\subsection{Eigenstress rate}

In the preceding section, the heterogeneous solid consisting of a uniform matrix $M$ and a single 
inclusion $\Omega$ with different pseudo-moduli, defined in Eq. (36a) and Eq. (36b), is homogenized by the introduction of the eigenvelocity gradient $\dot{\mathbf{F}}^{*}(\mathbf{X})$. The homogenization can be performed by the introduction of an eigen- stress rate $\dot{\mathbf{S}}^{N *}(\mathbf{X})$, instead. To this end, set

$\dot{\mathbf{S}}^{N *}(\mathbf{X})= \begin{cases}\mathbf{0} & \text { in } M \\ \dot{\mathbf{S}}^{N *} & \text { in } \Omega .\end{cases}$

For this alternative equivalent problem, the pseudo-modulus tensor is again uniform everywhere, including in $\Omega$. The corresponding velocity gradient and nominal stress rate fields are

$$
\begin{aligned}
\dot{\mathbf{F}}(\mathbf{X}) & =\dot{\mathbf{F}}^{0}+\dot{\mathbf{F}}^{d}(\mathbf{X}), \\
\dot{\mathbf{S}}^{N}(\mathbf{X}) & =\mathscr{\mathscr { F }}: \dot{\mathbf{F}}(\mathbf{X})+\dot{\mathbf{S}}^{N *}(\mathbf{X}) \\
& = \begin{cases}\mathscr{F}:\left(\dot{\mathbf{F}}^{0}+\dot{\mathbf{F}}^{d}(\mathbf{X})\right) & \text { in } M \\
\mathscr{F}:\left(\dot{\mathbf{F}}^{0}+\dot{\mathbf{F}}^{d}(\mathbf{X})\right)+\dot{\mathbf{S}}^{N *}(\mathbf{X}) & \text { in } \Omega .\end{cases}
\end{aligned}
$$

From Eq. (32c), the disturbance velocity gradient and the nominal stress rate must satisfy

$$
\dot{\mathbf{S}}^{N d}(\mathbf{X})=\mathscr{\mathscr { F }}: \dot{\mathbf{F}}^{d}(\mathbf{X})+\dot{\mathbf{S}}^{N *}(\mathbf{X}) \text { in } V,
$$

for the required eigenstress rate, $\dot{\mathbf{S}}^{N *}(\mathbf{X})$. In general, the nominal stress rate field $\dot{\mathbf{S}}^{N d}(\mathbf{X})$ can be expressed in terms of an integral operator acting on the corresponding eigenstress rate $\dot{\mathbf{S}}^{N *}(\mathbf{X})$. However, when $\Omega$ is ellipsoidal, and when the eigenvelocity gradient $\dot{\mathbf{F}}^{*}$ and hence the resulting disturbance velocity gradient $\dot{\mathbf{F}}^{d}$ are uniform in $\Omega$, then the corresponding eigenstress rate $\dot{\mathbf{S}}^{N *}$ and the associated disturbance nominal stress rate $\dot{\mathbf{S}}^{N d}$ are also uniform in $\Omega$. Therefore, a fourth-order tensor $\mathscr{T}^{\Omega}$, may be introduced such that

$\dot{\mathbf{S}}^{N d}=\mathscr{T}: \dot{\mathbf{S}}^{N *} \quad$ or $\quad \dot{\mathbf{S}}_{A i}^{N d}=\mathscr{T}_{A i j B}^{\Omega} \dot{S}_{B j}^{N *}$ in $\Omega$.

In general, tensor $\mathscr{T}^{\Omega}$ has no symmetries. It is conjugate to the generalized Eshelby tensor, $\mathscr{S}^{\Omega}$. Note from Eq. (38a), Eq. (38b), and Eqs. (41a)(41c) that the eigenvelocity gradient and the eigenstress rate are related by

$$
\begin{aligned}
& \dot{\mathbf{S}}^{N *}+\mathscr{F}: \dot{\mathbf{F}}^{*}=\mathbf{0}, \\
& \dot{\mathbf{F}}^{*}+\mathscr{G}: \dot{\mathbf{S}}^{N *}=\mathbf{0}
\end{aligned}
$$

or, in component form,

$\dot{S}_{A i}^{N *}+\mathscr{F}_{A i B j} \dot{F}_{j B}^{*}=0$,

$\dot{F}_{i A}^{*}+\mathscr{G}_{i A j B} \dot{S}_{B j}^{N *}=0$.

In view of Eqs. (30a)-(30c) and Eqs. (44a)(44d), expression (42) can be rewritten as

$\dot{\mathbf{F}}^{d}=\mathscr{G}: \dot{\mathbf{S}}^{N d}+\dot{\mathbf{F}}^{*}=\mathscr{G}:\left(\mathscr{T}^{\Omega}-\mathbf{1}^{(4)}\right): \dot{\mathbf{S}}^{N *}$ in $\Omega$

or, in component form,

$$
\begin{aligned}
\dot{F}_{i A}^{d} & =\mathscr{G}_{i A j B} \dot{S}_{B j}^{N d}+\dot{F}_{i A}^{*} \\
& =\mathscr{G}_{i A j B}\left(\mathscr{T}_{B j k C}^{\Omega}-\delta_{j k} \delta_{B C}\right) \dot{S}_{C k}^{N *} \text { in } \Omega .
\end{aligned}
$$

Hence,

$\mathscr{S}^{\Omega}: \dot{\mathbf{F}}^{*}=\mathscr{G}:\left(\mathscr{T}^{\Omega}-\mathbf{1}^{(4)}\right):\left(-\mathscr{\mathscr { F }}: \dot{\mathbf{F}}^{*}\right)$,

$\mathscr{T}^{\Omega}: \dot{\mathbf{S}}^{N *}=\mathscr{F}:\left(\mathscr{S}^{\Omega}-\mathbf{1}^{(4)}\right):\left(-\mathscr{G}: \dot{\mathbf{S}}^{N 0}\right)$.

Therefore, the tensors $\mathscr{S}^{\Omega}$ and $\mathscr{T}^{\Omega}$ satisfy

$\mathscr{S}^{\Omega}+\mathscr{G}: \mathscr{T}^{\Omega}: \mathscr{F}=\mathbf{1}^{(4)}$,

$\mathscr{T}^{\Omega}+\mathscr{\mathscr { F }}: \mathscr{S}^{\Omega}: \mathscr{G}=\mathbf{1}^{(4)}$.

In component form, these are

$\mathscr{S}_{i A B j}^{\Omega}+\mathscr{G}_{i A k C} \mathscr{T}_{C k l D^{\Omega}}^{\mathscr{F}}{ }_{D l B j}=\delta_{i j} \delta_{A B}$,

$\mathscr{T}_{A i j B}^{\Omega}+\mathscr{F}_{A i C k} \mathscr{S}_{k C D l}^{\Omega} \mathscr{G}_{l D j B}=\delta_{A B} \delta_{i j}$,

where all indices take on the values 1,2 , and 3, and repeated indices are summed.

\subsection{Consistency conditions}

For finite $V$, the eigenvelocity gradients or eigenstress rates necessary for homogenization are, in general, nonuniform in $\Omega$, even if $\Omega$ is ellipsoidal. Also, for a nonellipsoidal $\Omega$, the required eigenvelocity gradients or eigenstress rates are in general, variable in $\Omega$ (they are zero outside of $\Omega$ ), even if $V$ is unbounded. For the general case, the eigenvelocity gradient, $\dot{\mathbf{F}}^{*}(\mathbf{X})$, or the eigenstress rate, $\dot{\mathbf{S}}^{N *}(\mathbf{X})$, is defined by the so-called consistency conditions which require the resulting nominal stress rate field $\dot{\mathbf{S}}^{N}(\mathbf{X})$, or the velocity gradient field $\dot{\mathbf{F}}(\mathbf{X})$, to be the same under the applied overall loads, whether it is calculated through 
homogenization or directly by solving the corresponding boundary-value problem for the rate quantities in the original heterogeneous solid. Hence, the resulting nominal stress rate field in $\Omega$ becomes,

$$
\begin{aligned}
\dot{\mathbf{S}}^{N}(\mathbf{X}) & =\mathscr{F}^{\Omega}:\left\{\dot{\mathbf{F}}^{0}+\dot{\mathbf{F}}^{d}(\mathbf{X})\right\} \\
& =\mathscr{F}:\left\{\dot{\mathbf{F}}^{0}+\dot{\mathbf{F}}^{d}(\mathbf{X})-\dot{\mathbf{F}}^{*}(\mathbf{X})\right\} \text { in } \Omega,
\end{aligned}
$$

and the resulting velocity gradient field in $\Omega$ satisfies,

$$
\begin{aligned}
\dot{\mathbf{F}}(\mathbf{X}) & =\mathscr{G}^{\Omega}:\left\{\dot{\mathbf{S}}^{N 0}+\dot{\mathbf{S}}^{N d}(\mathbf{X})\right\} \\
& =\mathscr{G}:\left\{\dot{\mathbf{S}}^{N 0}+\dot{\mathbf{S}}^{N d}(\mathbf{X})-\dot{\mathbf{S}}^{N *}(\mathbf{X})\right\} \text { in } \Omega .
\end{aligned}
$$

It is noted that both Eqs. (47a) and (47b) are valid whether uniform tractions produced by $\dot{\mathbf{S}}^{N 0}$ or linear velocities produced by $\dot{\mathbf{F}}^{0}$ are prescribed on $\partial V$. If the overall nominal stress rate $\dot{\mathbf{S}}^{N 0}$ is given, then $\dot{\mathbf{F}}^{0}$ is defined by $\mathscr{G}: \dot{\mathbf{S}}^{N 0}$, whereas if the overall velocity gradient $\mathbf{F}^{0}$ is given, then $\dot{\mathbf{S}}^{N 0}$ is defined by $\mathscr{F}: \dot{\mathbf{F}}^{0}$.

Whether $V$ is bounded or not, and for any homogeneous inclusion $\Omega$ in a homogeneous matrix $M$ which satisfies Eqs. (36a) and (36b) consistency conditions (47a) and (47b) yield

$\dot{\mathbf{F}}^{0}+\dot{\mathbf{F}}^{d}(\mathbf{X})=\mathscr{C}^{\Omega}: \dot{\mathbf{F}}^{*}(\mathbf{X})$,

$\dot{\mathbf{S}}^{N 0}+\dot{\mathbf{S}}^{N d}(\mathbf{X})=\mathscr{D}^{\Omega}: \dot{\mathbf{S}}^{N *}(\mathbf{X})$ in $\Omega$,

where

$\mathscr{C}^{\Omega} \equiv\left(\mathscr{\mathscr { F }}-\mathscr{\mathscr { F }}^{\Omega}\right)^{-1}: \mathscr{\mathscr { F }}$,

$\mathscr{D}^{\Omega} \equiv\left(\mathscr{G}-\mathscr{G}^{\Omega}\right)^{-1}: \mathscr{G}$.

By definition, constant tensors $\mathscr{C}^{\Omega}$ and $\mathscr{D}^{\Omega}$ satisfy

$\mathscr{G}: \mathscr{\mathscr { F }}^{\Omega}=\mathbf{1}^{(4)}-\left(\mathscr{C}^{\Omega}\right)^{-1}$

or

$\mathscr{F}: \mathscr{G}^{\Omega}=\mathbf{1}^{(4)}-\left(\mathscr{D}^{\Omega}\right)^{-1}$.

In general, $\mathscr{C}^{\Omega}$ and $\mathscr{D}^{\Omega}$ do not have any symmetries, as can be seen from their components,

$\mathscr{C}_{A i B j}^{\Omega} \equiv\left(\delta_{i j} \delta_{A B}-\mathscr{G}_{i A k C} \mathscr{F}_{C k B j}^{\Omega}\right)^{-1}$,

$\mathscr{D}_{i A j B}^{\Omega} \equiv\left(\delta_{A B} \delta_{i j}-\mathscr{F}_{A i C k} \mathscr{G}_{k C j B}^{\Omega}\right)^{-1}$.

In the sequel, attention is confined to the case when $V$ is unbounded and $\Omega$ is ellipsoidal, so that the homogenization eigenvelocity gradient and eigenstress rate are both uniform in $\Omega$. When $V$ is unbounded, there is no distinction between the cases when the velocity gradient $\dot{\mathbf{F}}^{0}$ or the nominal stress rate $\dot{\mathbf{S}}^{N 0}$ is prescribed. Thus $\dot{\mathbf{F}}^{0}=$ $\mathscr{G}: \dot{\mathbf{S}}^{N 0}$ or $\dot{\mathbf{S}}^{N 0}=\mathscr{\mathscr { F }}: \dot{\mathbf{F}}^{0}$. Also, when, in addition, $\Omega$ is ellipsoidal, then $\dot{\mathbf{F}}^{d}, \dot{\mathbf{F}}^{*}, \dot{\mathbf{S}}^{N d}$, and $\dot{\mathbf{S}}^{N *}$ are all constant tensors in $\Omega$. Hence, for unbounded $V$ and ellipsoidal $\Omega$, substitution for $\dot{\mathbf{F}}^{d}$ in Eq. (48a) or for $\dot{\mathbf{S}}^{N d}$ in Eq. (48b) provides explicit expressions for the eigenvelocity gradient $\dot{\mathbf{F}}^{*}$ and eigenstress rate $\dot{\mathbf{S}}^{N *}$ which are necessary for homogenization,

$\dot{\mathbf{F}}^{*}=\left(\mathscr{C}^{\Omega}-\mathscr{S}^{\Omega}\right)^{-1}: \dot{\mathbf{F}}^{0}$,

$\dot{\mathbf{S}}^{N *}=\left(\mathscr{D}^{\Omega}-\mathscr{T}^{\Omega}\right)^{-1}: \dot{\mathbf{S}}^{N 0}$ in $\Omega$.

These and Eqs. (40a) and (40b) now lead to

$\dot{\mathbf{F}}=\dot{\mathbf{F}}^{0}+\dot{\mathbf{F}}^{d}=\mathscr{C}^{\Omega}:\left(\mathscr{C}^{\Omega}-\mathscr{S}^{\Omega}\right)^{-1}: \dot{\mathbf{F}}^{0}$,

$\dot{\mathbf{S}}^{N}=\dot{\mathbf{S}}^{N 0}+\dot{\mathbf{S}}^{N d}$

$$
=\mathscr{D}^{\Omega}:\left(\mathscr{D}^{\Omega}-\mathscr{T}^{\Omega}\right)^{-1}: \dot{\mathbf{S}}^{N 0} \text { in } \Omega .
$$

Note that the velocity gradient $\dot{\mathbf{F}}$ and the nominal stress rate $\dot{\mathbf{S}}^{N}$ in $\Omega$, given by Eqs. (51a) and (51b), are equivalent. From constitutive relations (30a)-(30c), substitution of Eqs. (49a) and (49b) into Eqs. (51a) and (51b) yields

$$
\begin{aligned}
\dot{\mathbf{S}}^{N} & =\mathscr{\mathscr { P }}^{\Omega}: \mathscr{C}^{\Omega}:\left(\mathscr{C}^{\Omega}-\mathscr{S}^{\Omega}\right)^{-1}: \dot{\mathscr{F}}^{0} \\
& =\left\{\mathscr{F}^{\Omega}:\left\{\mathbf{1}^{(4)}-\mathscr{S}^{\Omega}:\left(\mathbf{1}^{(4)}-\mathscr{G}: \mathscr{\mathscr { F }}^{\Omega}\right)\right\}^{-1}: \mathscr{G}\right\}: \dot{\mathbf{S}}^{N 0},
\end{aligned}
$$

$$
\begin{aligned}
\dot{\mathbf{F}} & =\mathscr{G}^{\Omega}: \mathscr{D}^{\Omega}:\left(\mathscr{D}^{\Omega}-\mathscr{T}^{\Omega}\right)^{-1}: \dot{\mathbf{S}}^{N 0} \\
& =\left\{\mathscr{G}^{\Omega}:\left\{\mathbf{1}^{(4)}-\mathscr{T}:\left(\mathbf{1}^{(4)}-\mathscr{\mathscr { F }}: \mathscr{G}^{\Omega}\right)\right\}^{-1}: \mathscr{F}\right\}: \dot{\mathbf{F}}^{0} .
\end{aligned}
$$

Noting identities (46c) and (46d), observe that the fourth-order tensors in the right-hand sides of Eqs. (52a) and (52b) become

$$
\begin{aligned}
\mathscr{F}^{\Omega}:\left\{\mathbf{1}^{(4)}-\mathscr{T}^{\Omega}:\left(\mathbf{1}^{(4)}-\mathscr{G}: \mathscr{\mathscr { P }}^{\Omega}\right)\right\}^{-1}: \mathscr{G} \\
\quad=\left\{\mathbf{1}^{(4)}-\mathscr{T}^{\Omega}:\left(\mathbf{1}^{(4)}-\mathscr{T}: \mathscr{G}^{\Omega}\right)\right\}^{-1}, \\
\mathscr{G}^{\Omega}:\left\{\mathbf{1}^{(4)}-\mathscr{T}^{\Omega}:\left(\mathbf{1}^{(4)}-\mathscr{\mathscr { P }}: \mathscr{G}^{\Omega}\right)\right\}^{-1}: \mathscr{\mathscr { F }} \\
\quad=\left\{\mathbf{1}^{(4)}-\mathscr{S}^{\Omega}:\left(\mathbf{1}^{(4)}-\mathscr{G}: \mathscr{F}^{\Omega}\right)\right\}^{-1} .
\end{aligned}
$$


When these are compared with Eqs. (51a) and (51b), the following equivalence relations ${ }^{14}$ between $\left(\mathscr{C}^{\Omega}, \mathscr{S}^{\Omega}\right)$ and $\left(\mathscr{D}^{\Omega}, \mathscr{T}^{\Omega}\right)$ are obtained:

$$
\begin{aligned}
& \mathscr{F}^{\Omega}: \mathscr{C}^{\Omega}:\left(\mathscr{C}^{\Omega}-\mathscr{S}^{\Omega}\right)^{-1}: \mathscr{G}=\mathscr{D}^{\Omega}:\left(\mathscr{D}^{\Omega}-\mathscr{T}^{\Omega}\right)^{-1}, \\
& \mathscr{G}^{\Omega}: \mathscr{D}:\left(\mathscr{D}^{\Omega}-\mathscr{T}^{\Omega}\right)^{-1}: \mathscr{\mathscr { I }}=\mathscr{C}^{\Omega}:\left(\mathscr{C}^{\Omega}-\mathscr{S}^{\Omega}\right)^{-1}
\end{aligned}
$$

\subsection{Concentration tensors}

Eqs. (51a) and (51b) yield the velocity gradient, $\dot{\mathbf{F}}$, and the nominal stress rate, $\dot{\mathbf{S}}^{N}$, in an ellipsoidal inclusion, $\Omega$, of pseudo-modulus tensor $\mathscr{F}^{\Omega}$, which is embedded in an unbounded homogeneous solid of pseudo-modulus tensor $\mathscr{\mathscr { P }}$, under farfield uniform data, $\dot{\mathbf{S}}^{N 0}=\mathscr{\mathscr { P }}: \dot{\mathbf{F}}^{0}$. These, thus, define the concentration tensors which relate the local fields in $\Omega$ to the farfield data, as follows:

$$
\begin{aligned}
& \dot{\mathbf{F}}=\mathscr{A}^{\Omega}: \dot{\mathbf{F}}^{0}, \\
& \dot{\mathbf{S}}^{N}=\mathscr{B}^{\Omega}: \dot{\mathbf{S}}^{N 0},
\end{aligned}
$$

where

$\mathscr{A}^{\Omega} \equiv \mathscr{C}^{\Omega}:\left(\mathscr{C}^{\Omega}-\mathscr{S}^{\Omega}\right)^{-1}$,

$\mathscr{B}^{\Omega} \equiv \mathscr{D}^{\Omega}:\left(\mathscr{D}^{\Omega}-\mathscr{T}^{\Omega}\right)^{-1}$

are the concentration tensors; here, $\mathscr{C}^{\Omega}$ and $\mathscr{D}^{\Omega}$ are defined by Eqs. (49a) and (49b). These tensors may be expressed as

$$
\begin{aligned}
& \mathscr{A}^{\Omega}=\left\{\mathbf{1}^{(4)}-\mathscr{P}^{\Omega}:\left(\mathscr{\mathscr { F }}-\mathscr{F}^{\Omega}\right)\right\}^{-1}, \\
& \mathscr{B}^{\Omega}=\left\{\mathbf{1}^{(4)}-\mathscr{Q}^{\Omega}:\left(\mathscr{G}-\mathscr{G}^{\Omega}\right)\right\}^{-1} .
\end{aligned}
$$

Here, in addition to Eqs. (49a) and (49b), the following notation is used:

$$
\begin{aligned}
& \mathscr{P}^{\Omega} \equiv \mathscr{S}^{\Omega}: \mathscr{G}, \\
& \mathscr{Q}^{\Omega} \equiv \mathscr{T}^{\Omega}: \mathscr{F} .
\end{aligned}
$$

\footnotetext{
${ }^{14}$ Except for the symmetries that exist in linear elasticity, but not in the present case, the above relations are identical to the corresponding relations in linear elasticity; see Nemat-Nasser and Hori (1993).
}

In component form, the concentration tensors become,

$$
\begin{aligned}
& \mathscr{A}_{i A j B}^{\Omega} \equiv\left\{\delta_{i j} \delta_{A B}-\mathscr{P}_{i A k C}^{\Omega}\left(\mathscr{F}_{C k B j}-\mathscr{F}_{C k B j}^{\Omega}\right)\right\}^{-1}, \\
& \left.\mathscr{B}_{A i B j}^{\Omega} \equiv \delta_{A B} \delta_{i j}-\mathscr{Q}_{A i C k}^{\Omega}\left(\mathscr{G}_{k C j B}-\mathscr{G}_{k C j B}^{\Omega}\right)\right\}^{-1},
\end{aligned}
$$

where,

$$
\begin{gathered}
\mathscr{P}_{i A j B}^{\Omega}=\mathscr{S}_{i A C k}^{\Omega} \mathscr{G}_{k C j B}^{\Omega}, \\
\mathscr{2}_{A i B j}^{\Omega}=\mathscr{T}_{A i k C}^{\Omega} \mathscr{\mathscr { F }}_{C k B j}^{\Omega} .
\end{gathered}
$$

Tensor $\mathscr{P}^{\Omega}$ (and hence $\mathscr{Q}^{\Omega}$ ) is obtained in Section 6.3 in terms of the Green function of the unbounded $V$ of pseudo-modulus tensor $\mathscr{F ^ { \prime }}$ (or, pseudo-compliance $\mathscr{G}$ ) and the aspect ratios of the ellipsoid $\Omega$. These tensors do not depend on the properties of $\Omega$. They are real-valued and can be computed as long as the operator $\mathscr{F}_{A i B j} \partial^{2}(\ldots) /\left(\partial X_{A} \partial X_{B}\right)$ is elliptic.

\section{Green's function and concentration tensors}

In Sections 3.2 and 3.4, the generalized Eshelby tensor, $\mathscr{S}^{\Omega}$, and its conjugate, $\mathscr{T}^{\Omega}$, are introduced for an infinitely extended homogeneous solid of pseudo-modulus and pseudo-compliance tensors, $\mathscr{F}$ and $\mathscr{G}$. These are then used to determine the constant velocity gradient and the nominal stress rate fields, $\dot{\mathbf{F}}$ and $\dot{\mathbf{S}}^{N}$, in an ellipsoidal subdomain $\Omega$, in which a uniform eigenvelocity gradient (or eigenstress rate) is distributed. As pointed out in Section 3.3, the generalized Eshelby tensor and its conjugate can be used to estimate the average velocity gradients and the nominal stress rates in an inclusion of pseudo-modulus and pseudo-compliance tensors, $\mathscr{\mathscr { F }}^{\Omega}$ and $\mathscr{G}^{\Omega}$, which is embedded in an unbounded uniform matrix. Under suitable settings, these tensors can be computed. Therefore, they provide an effective means for estimating the overall pseudo moduli of a heterogeneous solid.

In this section, an infinitely extended homogeneous solid is considered within a portion of which either eigenvelocity gradients or eigenstress rates are distributed (not necessarily uniformly). The Green function for the unbounded solid is then used to formulate the resulting velocity field in terms of two integral operators, $\mathscr{S}^{\infty}\left(\mathbf{X} ; \dot{\mathbf{F}}^{*}\right)$ and 
$\mathscr{T}^{\infty}\left(\mathbf{X} ; \dot{\mathbf{S}}^{N *}\right)$, where $\dot{\mathbf{F}}^{*}=\dot{\mathbf{F}}^{*}(\mathbf{X})$ and $\dot{\mathbf{S}}^{N *}=\dot{\mathbf{S}}^{N *}(\mathbf{X})$ are the corresponding prescribed eigenvelocity gradient and eigenstress rate. ${ }^{15}$ The integral operators reduce to tensor operators, when the distribution of eigenvelocity gradients or eigenstress rates is uniform and the domain $\Omega$ in which they are distributed is an isolated ellipsoid in an unbounded uniform medium, resulting in the generalized Eshelby tensor, $\mathscr{S}^{\Omega}$, and its conjugate, $\mathscr{T}^{\Omega}$. Equations are derived for these tensors in the general case of a matrix with an instantaneous pseudo-modulus which corresponds to complexvalued characteristics. Then a number of interesting properties of the generalized Eshelby tensor are examined, showing how certain results from linear elasticity can be generalized for application to finite deformation plasticity which involves full geometric and material nonlinearities. Many of these results are new. For example, it is shown how the double-inclusion problem is solved for the velocity gradient and the nominal stress rate imposed on the finite deformation and rotation of fully nonlinear elastoplastic heterogeneous solids. From this, the disturbances produced in the average velocity gradient, nominal stress rate, and other related quantities, by prescribed eigenvelocity gradients or eigenstress rates, are obtained. As before, throughout this section, quasi-static deformation is considered. All rate quantities are measured with respect to a monotonically changing parameter. Thus, the rate of change of any field represents its instantaneous quasi-static variation, from which its incremental change is calculated.

\subsection{Reciprocal relations}

As pointed out in Section 3.2, for a broad class of problems, the pseudo-modulus tensor $\mathscr{\mathscr { T }}$, with components $\mathscr{F}_{A i B j}$, is symmetric with respect to the

\footnotetext{
${ }^{15}$ Only the eigenvelocity gradient or the eigenstress rate, but not both, can be prescribed arbitrarily in a given region. Hence, when both eigenvelocity gradient and eigenstress rate are mentioned, either two separate problems are considered, or the two fields are mutually dependent. The intended alternative should be clear from the context
}

exchange of $A i$ and $B j$, i.e., the nine by nine matrix of $\mathscr{F}_{A i B j}$ has diagonal symmetry. Then, the reciprocal theorem of linear elasticity remains valid for rate problems.

To show this, consider a finite solid with volume $V$ and boundary $\partial V$, having such a modulus tensor, and let it be in equilibrium under two sets of boundary data, as follows:

$$
\begin{aligned}
& \boldsymbol{\nabla} \cdot \dot{\mathbf{S}}^{N(\alpha)}(\mathbf{X})+\dot{\mathbf{f}}^{(\alpha)}(\mathbf{X})=\mathbf{0} \text { in } V, \\
& \mathbf{N} \cdot \dot{\mathbf{S}}^{N(\alpha)}=\dot{\mathbf{T}}^{(\alpha)} \text { on } \partial V, \quad \alpha=1,2 .
\end{aligned}
$$

Note that these are two different problems, associated with the same solid. Now take the dot product of Eq. (55a) with $\dot{\mathbf{x}}^{(\beta)}$ and integrate the resulting expression over $V$ to obtain,

$$
\begin{aligned}
\int_{\partial V} \dot{\mathbf{x}}^{(\beta)} \dot{\mathbf{T}}^{(\alpha)} \mathrm{d} V+\int_{V} \dot{\mathbf{x}}^{(\beta)} \cdot \dot{\mathbf{f}}^{(\alpha)} \mathrm{d} V \\
\quad=\int_{V} \dot{\mathbf{F}}^{(\beta)}: \dot{\mathbf{S}}^{N(\alpha)} \mathrm{d} V=\int_{V} \dot{\mathbf{F}}^{(\beta)}: \mathscr{\mathscr { F }}: \dot{\mathbf{F}}^{(\alpha)} \mathrm{d} V,
\end{aligned}
$$

where the Gauss theorem, the boundary data (55b) and (30a) are used. Since the nine by nine matrix of the components of $\mathscr{F}$ is diagonally symmetric,

$\int_{V} \dot{\mathbf{F}}^{(\beta)}: \mathscr{\mathscr { F }}: \dot{\mathbf{F}}^{(\alpha)} \mathrm{d} V=\int_{V} \dot{\mathbf{F}}^{(\alpha)}: \mathscr{\mathscr { F }}: \dot{\mathbf{F}}^{(\beta)} \mathrm{d} V$,

and, hence,

$$
\begin{aligned}
\int_{\partial V} \dot{\mathbf{x}}^{(\beta)} \cdot \dot{\mathbf{T}}^{(\alpha)} \mathrm{d} V+\int_{V} \dot{\mathbf{x}}^{(\beta)} \cdot \dot{\mathbf{f}}^{(\alpha)} \mathrm{d} V \\
\quad=\int_{\partial V} \dot{\mathbf{x}}^{(\alpha)} \cdot \dot{\mathbf{T}}^{(\beta)} \mathrm{d} V+\int_{V} \dot{\mathbf{x}}^{(\alpha)} \cdot \dot{\mathbf{f}}^{(\beta)} \mathrm{d} V .
\end{aligned}
$$

From this result it follows that, in an unbounded solid, the displacement rate $\dot{\mathbf{x}}^{(1)}\left(\mathbf{X}^{(2)}\right)$ at point $\mathbf{X}^{(2)}$ due to a unit $\dot{\mathbf{f}}$ applied at point $\mathbf{X}^{(1)}$, is equal to the displacement rate $\dot{\mathbf{x}}^{(2)}\left(\mathbf{X}^{(1)}\right)$ at point $\mathbf{X}^{(1)}$ due to a unit $\dot{\mathbf{f}}$ applied at point $\mathbf{X}^{(2)}$, i.e.,

$\dot{\mathbf{x}}^{(1)}\left(\mathbf{X}^{(2)}\right)=\dot{\mathbf{x}}^{(2)}\left(\mathbf{X}^{(1)}\right)$.

Expressions (55e) and (55f) are reciprocal relations, valid when the pseudo-modulus tensor $\mathscr{\mathscr { F }}$, with components $\widetilde{F}_{A i B j}$, is symmetric with respect to the exchange of $A i$ and $B j$. 


\subsection{Green's function}

Consider an infinitely extended domain, denoted by $V^{\infty}$, consisting of a homogeneous solid of pseudo-modulus $\mathscr{\mathscr { F }}$ and pseudo-compliance $\mathscr{G}$. Formally, the Green function for this solid, $G_{j m}^{\infty}(\mathbf{X}, \mathbf{Y})$, is the vector-valued fundamental solution of the operator $\mathscr{L}_{i j} \equiv \mathscr{F}_{A i B j} \partial^{2}(\ldots) /\left(\partial X_{A} \partial X_{B}\right)$, defined by the following boundary-value problem:

$\mathscr{F}_{A i B j} G_{j m, A B}^{\infty}(\mathbf{X}, \mathbf{Y})+\delta(\mathbf{X}-\mathbf{Y}) \delta_{i m}=0$,

where $\delta(\mathbf{X}-\mathbf{Y})$ is the delta function, and

$G_{j m}^{\infty}(\mathbf{X}, \mathbf{Y}) \rightarrow 0 \quad$ as $|X| \rightarrow \infty$.

Note that, in the expression of the Green function, the first subscript denotes the corresponding component, and the second subscript is the direction of an applied unit "force-rate". Hence, $G_{j m}^{\infty}$ is the $\mathbf{e}_{j}$-component of the vector-valued function $\mathbf{G}^{\infty}(\mathbf{X}, \mathbf{Y})$, measured at point $\mathbf{X}$, due to the unit "force-rate" applied at point $\mathbf{Y}$ in the direction $\mathbf{e}_{m}$.

Since the domain $V^{\infty}$ is homogeneous and unbounded, only the difference between the $\mathbf{X}$-point (where the "displacement rate" is measured) and the Y-point (where the unit "force-rate" is applied) determines the Green function,

$\mathbf{G}^{\infty}(\mathbf{X}, \mathbf{Y})=\mathbf{G}^{\infty}(\mathbf{X}-\mathbf{Y})$.

The farfield condition (56b) now becomes

$\mathbf{G}^{\infty}(\mathbf{Z}) \rightarrow \mathbf{0}$ as $|\mathbf{Z}| \rightarrow \infty$.

The gradient of $\mathbf{G}^{\infty}$ also vanishes for large values of $\mathbf{Z}$, and hence the farfield velocity gradients and the nominal stress rates are zero. For an arbitrary finite domain $W$ within $V^{\infty}$ to be in equilibrium, the resultant traction rates on the surface $\partial W$ of $W$ must satisfy

$$
\begin{gathered}
\int_{\partial W} \mathbf{N} \cdot\left\{\mathscr{F}:\left(\nabla_{\mathbf{X}} \otimes \mathbf{G}^{\infty}(\mathbf{X}-\mathbf{Y})\right\} \mathrm{d} S_{\mathbf{X}}\right. \\
\quad= \begin{cases}-\mathbf{1}^{(2)} & \text { if } \mathbf{Y} \text { in } W \\
\mathbf{0} & \text { otherwise, }\end{cases}
\end{gathered}
$$

where $\mathbf{N}$ is the outer unit normal of $\partial W$, and subscript $\mathbf{X}$ stands for differentiation (integration) with respect to $\mathbf{X}$. Eq. (58b) is obtained with the aid of the Gauss theorem, by integrating the governing Eq. (56a) over $W$.
The Green function $\mathbf{G}^{\infty}$ has the following symmetry property, derived from the reciprocal theorem Eq. (55f).

$\mathbf{G}^{\infty}(\mathbf{Z})=\mathbf{G}^{\infty T}(-\mathbf{Z})$

or

$G_{i j}^{\infty}(\mathbf{Z})=G_{j i}^{\infty}(-\mathbf{Z})$.

From the governing Eqs. (56a) and (56b), $\mathbf{G}^{\infty}$ is an even function of $\mathbf{Z}$. Thus, in view of Eqs. (59a) and (59b),

$\mathbf{G}^{\infty}(\mathbf{Z})=\mathbf{G}^{\infty T}(\mathbf{Z})$

or

$G_{i j}^{\infty}(\mathbf{Z})=G_{j i}^{\infty}(\mathbf{Z})$.

\subsection{The body-force-rate problem}

Consider the velocity field $\dot{\mathbf{x}}(\mathbf{X})$ produced by distributed body-force rate, $\dot{\mathbf{f}}(\mathbf{X})$, which vanishes toward infinity. The boundary-value problem for $\dot{\mathbf{x}}$ is,

$\boldsymbol{\nabla} \cdot\left\{\mathscr{\mathscr { F }}:(\boldsymbol{\nabla} \otimes \dot{\mathbf{x}}(\mathbf{X}))^{\mathrm{T}}\right\}+\dot{\mathbf{f}}(\mathbf{X})=\mathbf{0} \quad \mathbf{X}$ in $V^{\infty}$,

with

$\dot{\mathbf{x}}(\mathbf{X}) \rightarrow \mathbf{0}$ as $|\mathbf{X}| \rightarrow \infty$.

In component form, Eq. (61a) becomes,

$\mathscr{F}_{A i B j} \dot{x}_{j, A B}+\dot{f}_{i}=0 \quad \mathbf{X}$ in $V^{\infty}$.

From linearity, the solution is given by integrating the Green function $\mathbf{G}^{\infty}$, weighted by the prescribed body force-rate $\dot{\mathbf{f}}$. Assuming that $\dot{\mathbf{f}}$ vanishes sufficiently quickly toward infinity, this gives,

$\dot{\mathbf{x}}(\mathbf{X})=\int_{V^{\infty}} \mathbf{G}^{\infty}(\mathbf{X}-\mathbf{Y}) \cdot \dot{\mathbf{f}}(\mathbf{Y}) \mathrm{d} V_{\mathbf{Y}}$,

or, in component form,

$\dot{x}_{i}(\mathbf{X})=\int_{V^{\infty}} G_{i m}^{\infty}(\mathbf{Y}-\mathbf{X}) \dot{f_{m}}(\mathbf{Y}) \mathrm{d} V_{\mathbf{Y}}$.

This is the unique solution of the boundary-value problem (61a)-(61c), valid for any $\dot{\mathbf{f}}$ (discontinuous or not) which renders the integral finite, provided that the ellipticity condition for the pseudo-modulus tensor, $\mathscr{\mathscr { F }}$, is satisfied. 
Suppose the body-force rate, $\dot{\mathbf{f}}$, is given by the divergence of a tensor field $\dot{\mathbf{S}}(\mathbf{X})$ which is sufficiently smooth in $W$ but suffers a jump to $\mathbf{0}$ across $\partial W$, i.e.,

$\dot{\mathbf{S}}(\mathbf{X})= \begin{cases}\dot{\mathbf{S}}(\mathbf{X}) \neq 0 & \mathbf{X} \text { in } W \\ \mathbf{0} & \text { otherwise },\end{cases}$

$\dot{\mathbf{f}}(\mathbf{X})=\nabla \cdot \dot{\mathbf{S}}(\mathbf{X}) \quad \mathbf{X}$ in $W$.

While $\mathbf{f}(\mathbf{X})$ is finite within $W$, it behaves as a delta function across $\partial W$. This behavior is represented by concentrated force-rates distributed within a thin layer about $\partial W$, representing the jump in $\dot{\mathbf{S}}(\mathbf{X})$ across this boundary; the overall effect, therefore, is represented by additional traction rates acting on $W$ over its boundary $\partial W$. Denote these traction rates by $[\dot{\mathbf{T}}](\mathbf{X})$ for $\mathbf{X}$ on $\partial W$, and obtain

$$
\begin{aligned}
& {[\dot{\mathbf{T}}](\mathbf{X}) \equiv \mathbf{N}(\mathbf{X}) \cdot[\dot{\mathbf{S}}](\mathbf{X})} \\
& =-\mathbf{N}(\mathbf{X}) \cdot\left\{\lim _{\mathbf{X}^{+} \rightarrow \mathbf{X}} \dot{\mathbf{S}}\left(\mathbf{X}^{+}\right)\right\}, \quad \mathbf{X} \text { on } \partial W,
\end{aligned}
$$

where the minus sign is due to the fact that the unit outer normal $\mathbf{N}$ points from the inside toward the outside of $W$, and $\mathbf{X}^{+}$is a point inside $W$. The resulting velocity field produced by body-force rate $\dot{\mathbf{f}}(\mathbf{X})$ distributed within $W$, and traction rates $[\dot{\mathbf{T}}](\mathbf{X})$ acting on $\partial W$, i.e., the velocity field corresponding to Eqs. (63b) and (63c), is given by

$$
\begin{aligned}
\dot{\mathbf{x}}(\mathbf{X})= & \int_{W} \mathbf{G}^{\infty T}(\mathbf{Y}-\mathbf{X}) \cdot \mathbf{f}(\mathbf{Y}) \mathrm{d} V_{\mathbf{Y}} \\
& +\int_{\partial W} \mathbf{G}^{\infty T}(\mathbf{Y}-\mathbf{X}) \cdot[\dot{\mathbf{T}}](\mathbf{Y}) \mathrm{d} S_{\mathbf{Y}},
\end{aligned}
$$

where the symmetry of $\mathbf{G}^{\infty}$, Eqs. (59a) and (59b), is used. In view of Eqs. (63b) and (63c), use the Gauss theorem to rewrite Eq. (64) as

$\dot{\mathbf{X}}(\mathbf{X})=-\int_{W}\left\{\nabla_{\mathbf{Y}} \otimes \mathbf{G}^{\infty T}(\mathbf{Y}-\mathbf{X})\right\}: \dot{\mathbf{S}}(\mathbf{Y}) \mathrm{d} V_{\mathbf{Y}}$,

or, in component form, ${ }^{16}$

\footnotetext{
${ }^{16}$ In view of the limited symmetries, the correct order of contractions must be preserved in the coordinate-independent notation.
}

$\dot{x}_{i}(\mathbf{X})=-\int_{W} G_{i k, A}^{\infty}(\mathbf{Y}-\mathbf{X}) \dot{S}_{A k}(\mathbf{Y}) \mathrm{d} V_{\mathbf{Y}}$,

where subscript $A$ following a comma denotes derivative with respect to $Y_{A}$.

\subsection{The eigenvelocity gradient or eigenstress rate problem}

Consider an eigenvelocity gradient field $\dot{\mathbf{F}}^{*}$ (or eigenstress rate field $\dot{\mathbf{S}}^{N *}$ ) prescribed ${ }^{17}$ in $W$ and vanishing identically outside of $W$,

$\dot{\mathbf{F}}^{*}(\mathbf{X})= \begin{cases}\dot{\mathbf{F}}^{*}(\mathbf{X}) & \mathbf{X} \text { in } W \\ \mathbf{0} & \text { otherwise }\end{cases}$

or

$\dot{\mathbf{S}}^{N *}(X)= \begin{cases}\dot{\mathbf{S}}^{N *}(\mathbf{X}) & \mathbf{X} \text { in } W \\ \mathbf{0} & \text { otherwise. }\end{cases}$

Let $\dot{\mathbf{x}}^{v}$ (let $\dot{\mathbf{x}}^{s}$ ) be the velocity field produced by $\dot{\mathbf{F}}^{*}$ (by $\dot{\mathbf{S}}^{N *}$ ) and denote by $\dot{\mathbf{F}}^{v}$ and $\dot{\mathbf{S}}^{N v}$ (by $\dot{\mathbf{F}}^{s}$ and $\dot{\mathbf{S}}^{N s}$ ) the corressponding velocity gradient and nominal stress rate fields. These velocity gradient and nominal stress rate fields satisfy

$\dot{\mathbf{F}}^{v}=\left(\boldsymbol{\nabla} \otimes \dot{\mathbf{x}}^{v}\right)^{T}$,

$\dot{\mathbf{S}}^{N v}=\mathscr{\mathscr { F }}: \dot{\mathbf{F}}^{v}-\mathscr{\mathscr { F }}: \dot{\mathbf{F}}^{*}$,

for the first problem, and

$\dot{\mathbf{F}}^{s}=\left(\boldsymbol{\nabla} \otimes \dot{\mathbf{x}}^{s}\right)^{T}$,

$\dot{\mathbf{S}}^{N s}=\mathscr{F}: \dot{\mathbf{F}}^{s}+\dot{\mathbf{S}}^{N *}$,

for the second problem; see Section 3.

The divergence of the eigenvelocity gradient $\dot{\mathbf{F}}^{*}$ and eigenstress rate $\dot{\mathbf{S}}^{N *}$ can be regarded as equivalent body-force rates. Indeed, direct substitution of the constitutive relations (67b) and (67d) into the equations of equilibrium yields

$\boldsymbol{\nabla} \cdot \dot{\mathbf{S}}^{N v}=\boldsymbol{\nabla} \cdot\left\{\mathscr{F}:\left(\boldsymbol{\nabla} \otimes \dot{\mathbf{x}}^{v}\right)^{T}\right\}+\nabla \cdot\left(-\mathscr{\mathscr { F }}: \dot{\mathbf{F}}^{*}\right)=\mathbf{0}$

\footnotetext{
${ }^{17}$ These are two separate problems which are being examined simultaneously.
} 
and

$\boldsymbol{\nabla} \cdot \dot{\mathbf{S}}^{N s}=\boldsymbol{\nabla} \cdot\left\{\mathscr{\mathscr { F }}:\left(\boldsymbol{\nabla} \otimes \dot{\mathbf{x}}^{S}\right)^{T}\right\}+\nabla \cdot \dot{\mathbf{S}}^{N *}=\mathbf{0}$.

The divergence of $-\mathscr{F}: \dot{F}^{*}$ (of $\dot{\mathbf{S}}^{N *}$ ) appears like a body-force rate in the governing equations for the velocity field $\dot{\mathbf{x}}^{v}$ (field $\dot{\mathbf{x}}^{s}$ ); see Eq. (61a). Hence, the velocity field $\dot{\mathbf{x}}^{v}$ (field $\dot{\mathbf{x}}^{s}$ ) may be expressed in terms of the Green function $\mathbf{G}^{\infty}$, as

$\dot{\mathbf{x}}^{v}(\mathbf{X})=\int_{W}\left\{\nabla_{\mathbf{Y}} \otimes \mathbf{G}^{\infty T}(\mathbf{Y}-\mathbf{X})\right\}:\left\{\mathscr{\mathscr { F }}: \dot{\mathbf{F}}^{*}(\mathbf{Y})\right\} \mathrm{d} V_{\mathbf{Y}}$

and

$\dot{\mathbf{x}}^{s}(\mathbf{X})=-\int_{W}\left\{\nabla_{\mathbf{Y}} \otimes \mathbf{G}^{\infty T}(\mathbf{Y}-\mathbf{X})\right\}: \dot{\mathbf{S}}^{N *}(\mathbf{Y}) \mathrm{d} V_{\mathbf{Y}}$

or, in component form,

$\dot{x}_{i}^{v}(\mathbf{X})=\int_{W} G_{i k, A}^{\infty}(\mathbf{Y}-\mathbf{X}) \mathscr{F}_{A k B j} \dot{F}_{j B}^{*}(\mathbf{Y}) \mathrm{d} V_{\mathbf{Y}}$

and

$\dot{x}_{i}^{s}(\mathbf{X})=-\int_{W} G_{i k, A}^{\infty}(\mathbf{Y}-\mathbf{X}) \dot{S}_{A k}^{N *}(\mathbf{Y}) \mathrm{d} V_{\mathbf{Y}}$,

where comma followed by the subscript $A$ denotes differentiation with respect to $Y_{A}$. The velocity field $\dot{\mathbf{x}}^{v}$ (field $\dot{\mathbf{x}}^{s}$ ) satisfies $\dot{\mathbf{x}}^{v}(\mathbf{Z}) \rightarrow \mathbf{0},\left(\dot{\mathbf{x}}^{s}(\mathbf{Z}) \rightarrow \mathbf{0}\right.$ ), as $|\mathbf{Z}| \rightarrow \infty$, and is continuous across $\partial W$.

In general, the velocity gradient and nominal stress rate fields, $\dot{\mathbf{F}}^{v}$ and $\dot{\mathbf{S}}^{N v}$, (fields $\dot{\mathbf{F}}^{s}$ and $\dot{\mathbf{S}}^{N s}$ ) are discontinuous across surfaces where the eigenvelocity gradient (eigenstress rate) admits finite discontinuities; the velocity field $\dot{\mathbf{x}}^{v}$ (field $\dot{\mathbf{x}}^{s}$ ) is, of course, continuous, as are the traction rates $\mathbf{N} \cdot \dot{\mathbf{S}}^{N v}$ (traction-rates $\mathbf{N} \cdot \dot{\mathbf{S}}^{N s}$ ).

Using the velocity gradient and the constitutive relations given by Eqs. (67a)-(67f), define the integral operator $\mathscr{S}^{\infty}$ (operator $\mathscr{T}^{\infty}$ ) which determines $\dot{\mathbf{F}}^{v}$ (determines $\left.\dot{\mathbf{S}}^{N s}\right)$, in terms of $\dot{\mathbf{F}}^{*}\left(\right.$ of $\left.\dot{\mathbf{S}}^{N *}\right)$, as follows:

$\mathscr{S}^{\infty}\left(\mathbf{X} ; \dot{\mathbf{F}}^{*}\right) \equiv \int_{W} \Gamma^{\infty}(\mathbf{Y}-\mathbf{X}): \mathscr{\mathscr { F }}: \dot{\mathbf{F}}^{*}(\mathbf{Y}) \mathrm{d} V_{\mathbf{Y}}$

$$
\begin{aligned}
& \mathscr{T}^{\infty}\left(\mathbf{X} ; \dot{\mathbf{S}}^{N *}\right) \\
& \equiv-\mathscr{\mathscr { F }}:\left\{\int_{W} \Gamma^{\infty}(\mathbf{Y}-\mathbf{X}): \dot{\mathbf{S}}^{N *}(\mathbf{Y}) \mathrm{d} V_{\mathbf{Y}}\right\}+\dot{\mathbf{S}}^{N *}(\mathbf{X}),
\end{aligned}
$$

or, in component form,

$\mathscr{S}_{i A}^{\infty}\left(\mathbf{X} ; \dot{\mathbf{F}}^{*}\right) \equiv \int_{W} \Gamma_{i A m C}^{\infty}(\mathbf{Y}-\mathbf{X}) \mathscr{\mathscr { F }}_{C m B l} \dot{F}_{l B}^{*}(\mathbf{Y}) \mathrm{d} V_{\mathbf{Y}}$

$$
\begin{aligned}
\mathscr{T}_{A i}^{\infty}\left(\mathbf{X} ; \dot{\mathbf{S}}^{N *}\right) \equiv & -\mathscr{\mathscr { F }}_{A i B j}\left\{\int_{W} \Gamma_{j B k C}^{\infty}(\mathbf{Y}-\mathbf{X})\right. \\
& \left.\times \dot{S}_{C k}^{N *}(\mathbf{Y}) \mathrm{d} V_{\mathbf{Y}}\right\}+\dot{S}_{A i}^{N *}(\mathbf{X}) .
\end{aligned}
$$

In Eqs. (69a)-(69d), the tensor field $\boldsymbol{\Gamma}^{\infty}(\mathbf{Y}-\mathbf{X})$ is defined by

$$
\begin{aligned}
& \Gamma_{i A j B}^{\infty}(\mathbf{Y}-\mathbf{X}) \\
& \quad \equiv-\frac{1}{2}\left\{G_{i j, A B}^{\infty}(\mathbf{Y}-\mathbf{X})+G_{j i, B A}^{\infty}(\mathbf{Y}-\mathbf{X})\right\},
\end{aligned}
$$

where comma followed by the subscripts $A$ and $B$ denotes differentiation with respect to $Y_{A}$ and $Y_{B}$ or $X_{A}$ and $X_{B}$. In view of the symmetries of the Green function, Eq. (60a) and Eq. (60b), the tensor field $\Gamma_{i A j B}^{\infty}$ has the following symmetries:

$\Gamma_{i A j B}^{\infty}=\Gamma_{j A i B}^{\infty}=\Gamma_{j B i A}^{\infty}=\Gamma_{i B j A}^{\infty}$.

Hence, while $\mathscr{F}_{i A j B}$, in general, may have, at most, 45 distinct components, $\Gamma_{i A j B}^{\infty}$ has, at most, only 36 distinct ${ }^{18}$ components. In Eq. (56a), which defines the Green function, only the following combination of the components of the pseudo-modulus tensor is involved: $\left(\mathscr{F}_{A i B j}+\mathscr{F}_{B i A j}\right) / 2$. In light of the assumed symmetry with respect to $A i$ and $B j$, it is seen that only 36 distinct combinations of the components of $\mathscr{F}_{A i B j}$ enter the solution of the Green function for an unbounded domain. This combination is, $\left(\mathscr{F}_{A i B j}+\mathscr{\mathscr { F }}_{B i A j}+\mathscr{F}_{A j B i}+\mathscr{F}_{B j A i}\right) / 4$.

\footnotetext{
${ }^{18}$ There are six distinct components for each fixed pair of $A$ and $B$, or for each fixed pair of $i$ and $j$. Hence, there are only 36 possible distinct components for $\Gamma_{i A j B}^{\infty}, i, j, A, B=1,2,3$.
} 
When the eigenfields $\dot{\mathbf{F}}^{*}$ and $\dot{\mathbf{S}}^{N *}$ correspond to each other in the sense that

$\dot{\mathbf{S}}^{N *}(\mathbf{X})=-\mathscr{F}: \dot{\mathbf{F}}^{*}(\mathbf{X})$

or

$\dot{\mathbf{F}}^{*}(\mathbf{X})=-\mathscr{G}: \dot{\mathbf{S}}^{N *}(\mathbf{X}) \quad \mathbf{X}$ in $W$,

then the resulting velocity fields $\dot{\mathbf{x}}^{v}$ and $\dot{\mathbf{x}}^{s}$ are the same, as are the resulting velocity gradient and nominal stress rate fields. Then Eq. (67a) and Eq. (67c) agree, and

$\dot{\mathbf{S}}^{N v}=\mathscr{\mathscr { P }}:\left(\dot{\mathbf{F}}^{v}-\dot{\mathbf{F}}^{*}\right)=\mathscr{F}: \dot{\mathbf{F}}^{s}+\dot{\mathbf{S}}^{N *}=\dot{\mathbf{S}}^{N s}$.

Therefore, the integral operators $\mathscr{S}^{\infty}$ and $\mathscr{T}^{\infty}$ satisfy

$\mathscr{T}^{\infty}\left(\mathbf{X} ;-\mathscr{\mathscr { F }}: \dot{\mathbf{F}}^{*}\right)=\mathscr{\mathscr { F }}:\left\{\mathscr{S}^{\infty}\left(\mathbf{X} ; \dot{\mathbf{F}}^{*}\right)-\dot{\mathbf{F}}^{*}(\mathbf{X})\right\}$

$\mathscr{S}^{\infty}\left(\mathbf{X} ;-\mathscr{G}: \dot{\mathbf{S}}^{N *}\right)=\mathscr{G}:\left\{\mathscr{T}^{\infty}\left(\mathbf{X} ; \dot{\mathbf{S}}^{N *}\right)-\dot{\mathbf{S}}^{N *}(\mathbf{X})\right\}$,

provided that $\dot{\mathbf{S}}^{N *}$ and $\dot{\mathbf{F}}^{*}$ are related by Eqs. (70a) and (70b). Eqs. (72a) and (72b) are the equivalence relations between the integral operators $\mathscr{S}^{\infty}$ and $\mathscr{T}^{\infty}$.

\subsection{Generalized Eshelby tensor and its conjugate}

In the preceding section, the integral operator $\mathscr{S}_{i A}^{\infty}\left(\mathbf{X} ; \dot{\mathbf{F}}^{*}\right)$ and its conjugate $\mathscr{T}_{A i}^{\infty}\left(\mathbf{X} ; \dot{\mathbf{S}}^{N *}\right)$, are expressed in terms of the tensor field $\Gamma_{j B k C}^{\infty}(\mathbf{Y}-\mathbf{X})$ which is given by the gradient of the Green function $G_{i j}^{\infty}(\mathbf{Z})$ of the unbounded domain $V^{\infty}$; see Eqs. (69a)-(69c) and Eqs. (69b)-(69d). The integral of $\Gamma_{j B k C}^{\infty}(\mathbf{Y}-\mathbf{X})$ over an ellipsoidal domain $\Omega$ turns out to be constant, as shown in Section 6.3. From this, the generalized Eshelby tensor $\mathscr{P}^{\Omega}$ and its conjugate $\mathscr{T}^{\Omega}$ are obtained.

Define the tensor field

$\mathscr{P}_{i A j B}(\mathbf{X} ; W) \equiv \int_{W} \Gamma_{i A j B}^{\infty}(\mathbf{Y}-\mathbf{X}) \mathrm{d} V_{\mathbf{Y}}$.

In terms of $\mathscr{P}_{i A j B}(\mathbf{X} ; W)$, the generalized Eshelby tensor $\mathscr{S}^{\Omega}$ for an ellipsoidal domain is given by,

$\mathscr{S}_{i A B j}^{\Omega}=\mathscr{P}_{i A k C}^{\Omega} \tilde{F}_{C k B j}$,

where
$\mathscr{P}_{i A j B}(\mathbf{X} ; \Omega) \equiv \mathscr{P}_{i A j B}^{\Omega}=$ constant $\quad$ for $\mathbf{X}$ in $\Omega ; \quad(73 \mathrm{c})$ see (54c,d,g,h) and Eqs. (69a)-(69f). As pointed out before, there are, at most, 36 distinct components for $\mathscr{P}_{i A j B}^{\Omega}$; see Eq. (69f). From the symmetry $\mathscr{P}_{i A j B}^{\Omega}=\mathscr{P}_{j B i A}^{\Omega}$, it follows that the generalized Eshelby tensor satisfies the following two symmetry properties:

$\mathscr{S}_{i A B j}^{\Omega} \mathscr{G}_{j B k C}=\mathscr{S}_{k C B j}^{\Omega} \mathscr{G}_{j B i A}\left(=\mathscr{P}_{i A k C}^{\Omega}\right)$

and

$\mathscr{\mathscr { F }}_{A i C k} \mathscr{S}_{k C B j}^{\Omega}=\mathscr{\mathscr { F }}_{B j C k} \mathscr{S}_{k C A i}^{\Omega}\left(=\mathscr{\mathscr { F }}_{B j C k} \mathscr{P}_{k C l D}^{\Omega} \mathscr{\mathscr { F }}_{D l A i}\right)$.

Consider now the integral operator $\mathscr{T}^{\infty}\left(\mathbf{X} ; \dot{\mathbf{S}}^{N *}\right)$ given by Eq. (69b), and using the equivalence relations (72a), define the tensor field $\mathscr{T}_{A i j B}^{\infty}(\mathbf{X} ; W)$ for a finite domain $W$ embedded in an unbounded domain $V^{\infty}$ of the uniform modulus tensor $\mathscr{F}$, as follows:

$$
\begin{aligned}
& \mathscr{T}_{A i j B}^{\infty}(\mathbf{X} ; W) \\
& \quad \equiv-\mathscr{F}_{A i C k} \int_{W} \Gamma_{k C j B}^{\infty}(\mathbf{Y}-\mathbf{X}) \mathrm{d} V_{\mathbf{Y}}+H(\mathbf{X} ; W) \delta_{A B} \delta_{i j}
\end{aligned}
$$

which, in terms of $\mathscr{P}_{A i B j}(\mathbf{X} ; W)$, becomes

$$
\begin{aligned}
& \mathscr{T}_{A i j B}^{\infty}(\mathbf{X} ; W) \\
& \quad=-\mathscr{F}_{A i C k} \mathscr{P}_{k C j B}(\mathbf{X} ; W)+H(\mathbf{X} ; W) \delta_{A B} \delta_{i j},
\end{aligned}
$$

where $H(\mathbf{X} ; W)$ is the Heaviside step function, having the value 1 for $\mathbf{X}$ is in $W$, and 0 otherwise.

When $W$ is an ellipsoid, $\Omega, \mathscr{T}^{\infty}(\mathbf{X} ; W)$ with components $\mathscr{T}_{A i i B}^{\infty}(\mathbf{X} ; \Omega)$, is constant for $\mathbf{X}$ in $\Omega$. It is then denoted by $\mathscr{T}^{\Omega}$, or in component form, by $\mathscr{T}_{A i j B}^{\Omega}$. Indeed, from (46d),

$\mathscr{T}_{A i j B}^{\Omega}=-\mathscr{F}_{A i C k} \mathscr{P}_{k C j B}^{\Omega}+\delta_{A B} \delta_{i j}$.

The tensor $\mathscr{T}_{A i j B}^{\Omega}$ is conjugate to the generalized Eshelby tensor $\mathscr{S}_{i A B j}^{\Omega}$. The equivalence relations between $\mathscr{S}_{i A B j}^{\Omega}$ and $\mathscr{T}_{A i j B}^{\Omega}$ are given by Eqs. (46a)(46f). From the symmetry $\mathscr{P}_{i A j B}^{\Omega}=\mathscr{P}_{j B i A}^{\Omega}$, it follows that the generalized conjugate Eshelby tensor satisfies the following two symmetry properties:

$\mathscr{G}_{i A k C} \mathscr{T}_{C k j B}^{\Omega}=\mathscr{G}_{j B k C}^{\Omega} \mathscr{T}_{C k i A}^{\Omega}$,

$\mathscr{T}_{A i k C}^{\Omega} \mathscr{F}_{C k B j}=\mathscr{T}_{B j k C}^{\Omega} \mathscr{F}_{C k A i}$. 


\section{Average quantities}

For an ellipsoidal region, $\Omega$, the tensor field $\mathscr{P}(\mathbf{X} ; \Omega)$ is constant when $\mathbf{X}$ is in $\Omega$. This leads to a number of interesting exact results, similar to those for linearly elastic solids; see Nemat-Nasser and Hori (1993), Section 11. The similarity, however, is only in the form of the expressions. Therefore, the differences must be carefully noted. In the present work, the focus is on finite inelastic deformations of solids, involving large strains, rotations, strain rates, and spins. The instantaneous pseudo-modulus and pseudo-compliance tensors, i.e., $\mathscr{F}$ and $\mathscr{G}$, display minimal symmetries, and do not possess the properties that generally exist in linear elasticity. Moreover, in general, the results for the finite deformation case are valid only when appropriate kinematical and dynamical variables are used. As is evident from the results of the preceding sections, the velocity gradient and the nominal stress rate, $\dot{\mathbf{F}}(\mathbf{X})$ and $\dot{\mathbf{S}}^{N}$, are indeed suitable quantities for this purpose. The reference configuration can be arbitrary. Other strain rate and stress rate tensors do not, in general, have similar characteristics, and, hence, may not lead to similar simple and powerful results. On the other hand, no such distinction is necessary in linear elasticity.

\subsection{Generalized double-inclusion problem}

Consider an arbitrary finite region $W$ in an unbounded domain $V^{\infty}$ of pseudo-modulus tensor $\mathscr{F}$. The region $W$ may consist of several disconnected subregions, say, $W_{\alpha}, \alpha=1,2, \ldots, n$, of arbitrary shape.

Suppose that an arbitrary eigenvelocity gradient field, $\dot{\mathbf{F}}^{*}(\mathbf{X})$, is distributed in $W$. Let $\Omega$ be an arbitrary ellipsoidal domain in $V^{\infty}$, such that $W$ is totally contained within $\Omega$; see Fig. 2 . Then, the average velocity gradient and the corresponding average nominal stress rate, taken over domain $\Omega$, are completely determined by the generalized Eshelby tensor for $\Omega$.

Indeed, denoting the average value of $\dot{\mathbf{F}}^{*}(\mathbf{X})$ over $W$ by $\left\langle\dot{\mathbf{F}}^{*}\right\rangle_{W}$, and those of $\mathbf{\mathbf { F }}(\mathbf{X})$ and $\dot{\mathbf{S}}^{N}$ over $\Omega$ by $\langle\dot{\mathbf{F}}\rangle_{\Omega}$ and $\left\langle\dot{\mathbf{S}}^{N}\right\rangle_{\Omega}$, respectively, obtain

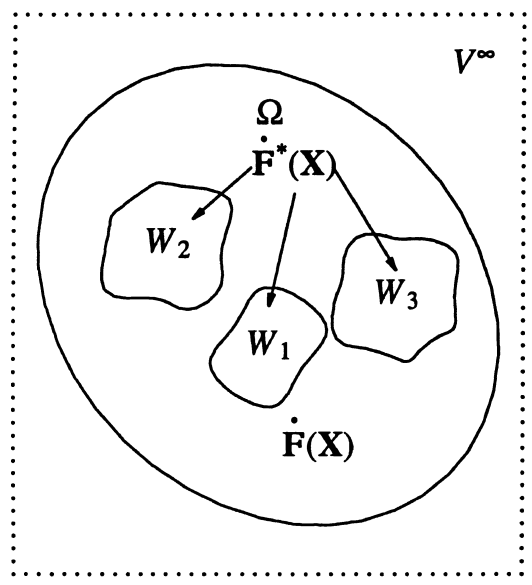

Fig. 2. An ellipsoidal $\Omega$ in an unbounded uniform $V^{\infty}$, contains $W=W_{1}+W_{2}+W_{3}$; arbitrary eigenvelocity gradients $\dot{\mathbf{F}}^{*}(\mathbf{X})$ are distributed within $W$.

$\langle\dot{\mathbf{F}}\rangle_{\Omega}=\frac{W}{\Omega} \mathscr{S}^{\Omega}:\left\langle\dot{\mathbf{F}}^{*}\right\rangle_{W}$,

$\langle\dot{\mathbf{S}}\rangle_{\Omega}=\frac{W}{\Omega} \mathscr{\mathscr { F }}:\left(\mathscr{S}^{\Omega}-\mathbf{1}^{(4)}\right):\left\langle\dot{\mathbf{F}}^{*}\right\rangle_{W}$

or, in component form,

$\left\langle\dot{F}_{i A}\right\rangle_{\Omega}=\frac{W}{\Omega} \mathscr{S}_{i A B j}^{\Omega}\left\langle\dot{F}_{j B}^{*}\right\rangle_{W}$

$\dot{S}_{A i}^{N}=\frac{W}{\Omega} \mathscr{F}_{A i B j}\left(\mathscr{S}_{j B C k}-\delta_{j k} \delta_{B C}\right)\left\langle\dot{F}_{k C}^{*}\right\rangle_{W}$,

where $W$ and $\Omega$ stand for their volumes.

The proof follows from the properties of the tensor field $\mathscr{P}_{A i B j}(\mathbf{X} ; W)$. For any eigenvelocity gradient $\dot{\mathbf{F}}^{*}(\mathbf{X})$ distributed in the finite domain $W$ within an unbounded uniform region $V^{\infty}$, the resulting velocity gradient field is

$\dot{F}_{i A}(\mathbf{X})=\int_{W} \Gamma_{i A j B}^{\infty}(\mathbf{Y}-\mathbf{X}) \mathscr{F}_{B j C k} \dot{F}_{k C}^{*}(\mathbf{Y}) \mathrm{d} V_{\mathbf{Y}}$

Therefore, the average velocity gradient in the ellipsoidal domain $\Omega$, becomes

$$
\begin{aligned}
& \left\langle\dot{F}_{i A}\right\rangle_{\Omega} \\
& \quad=\frac{1}{\Omega} \iint_{\Omega}\left\{\Gamma_{W}^{\infty}{ }_{i A j B}(\mathbf{Y}-\mathbf{X}) \mathscr{F}_{B j C k} \dot{F}_{k C}^{*}(\mathbf{Y}) \mathrm{d} V_{\mathbf{Y}}\right\} \mathrm{d} V_{\mathbf{X}},
\end{aligned}
$$


where $\mathbf{X}$ and $\mathbf{Y}$ are in $\Omega$ and $W$, respectively. Since $W$ and $\Omega$ do not intersect, the integrand $\Gamma_{i A j B}^{\infty}(\mathbf{X}-$ $\mathbf{Y})$ in Eq. (78a) is not singular. Hence, with the minimal assumption of integrability, the order of integration can be changed,

$$
\begin{aligned}
& \int_{\Omega}\left\{\int_{W} \Gamma_{i A j B}^{\infty}(\mathbf{Y}-\mathbf{X}) \mathscr{F}_{B j C k} \dot{F}_{k C}^{*}(\mathbf{Y}) \mathrm{d} V_{\mathbf{Y}}\right\} \mathrm{d} V_{\mathbf{X}} \\
& =\int_{W}\left\{\int_{\Omega} \Gamma_{i A j B}^{\infty}(\mathbf{Y}-\mathbf{X}) \mathrm{d} V_{\mathbf{X}}\right\} \mathscr{F}_{B j C k} \dot{F}_{k C}^{*}(\mathbf{Y}) \mathrm{d} V_{\mathbf{Y}} .
\end{aligned}
$$

The inner integral in the right-hand side is given by

$$
\int_{W} \Gamma_{i A j B}^{\infty}(\mathbf{Y}-\mathbf{X}) \mathrm{d} V_{\mathbf{X}}=\mathscr{P}_{i A j B}(\mathbf{Y} ; \Omega) \equiv \mathscr{P}_{i A j B}^{\Omega}
$$

which is constant for ellipsoidal $\Omega$, since $\mathbf{Y}$ is in $W \subset \Omega$. Therefore, in view of Eq. (73b), Eq. (75a) and Eq. (75b) is obtained. Eqs. (76a) and (76b) then follow by definition; e.g., use Eq. (42) and average over $\Omega$.

The remarkable exact results Eq. (75a) and Eq. (75b) and Eq. (76a) and Eq. (76b) are valid for any finite $W$, containing any arbitrary eigenvelocity gradient (transformation velocity gradient) $\dot{\mathbf{F}}^{*}$, which renders the integrals finite and permits the interchange of the order of integration. They can be used to homogenize a heterogeneous solid and to obtain the corresponding overall pseudo-moduli.

As an application of the general results Eq. (75a) and Eq. (75b) and Eq. (76a) and Eq. (76b), consider an ellipsoid $\Omega_{1}$ within another ellipsoid $\Omega_{2}$, embedded in a uniform unbounded domain $V^{\infty}$ of pseudo-modulus tensor $\mathscr{F}$; see Fig. 3. Let $\Omega_{1}$ contain an arbitrary region $W$, in which an arbitrary eigenvelocity gradient $\dot{\mathbf{F}}^{*}(\mathbf{X})$ is distributed. Under similar minimal assumptions, and using a similar procedure which produced Eq. (75a) and Eq. (75b), it can easily be shown that the average value of the velocity gradient over the annulus $\Omega_{2}-\Omega_{1}$, is given by

$$
\langle\dot{\mathbf{F}}\rangle_{\Omega_{2}-\Omega_{1}}=\frac{W}{\Omega_{2}-\Omega_{1}}\left\{\mathscr{S}\left(\Omega_{2}\right)-\mathscr{S}\left(\Omega_{1}\right)\right\}:\left\langle\dot{\mathbf{F}}^{*}\right\rangle_{W}
$$

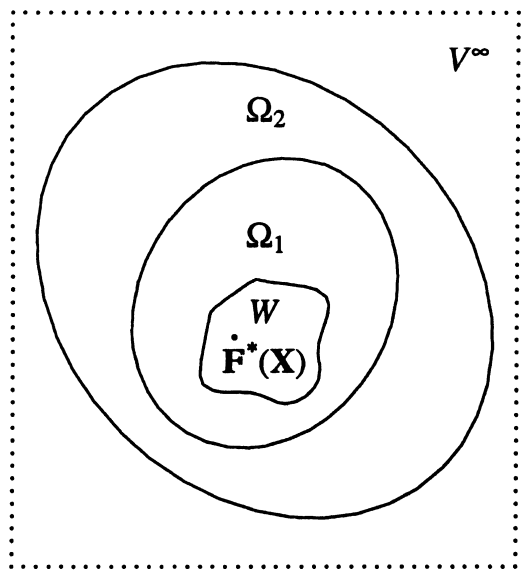

Fig. 3. An ellipsoidal $\Omega_{2}$ in an unbounded uniform $V^{\infty}$, contains an ellipsoidal $\Omega_{1}$ which contains an arbitrary region $W$; arbitrary eigenvelocity gradients $\dot{\mathbf{F}}^{*}(\mathbf{X})$ are distributed within $W$.

or, in component form, by

$$
\left\langle\dot{F}_{i A}\right\rangle_{\Omega_{2}-\Omega_{1}}=\frac{W}{\Omega_{2}-\Omega_{1}}\left\{\mathscr{S}_{i A B j}\left(\Omega_{2}\right)-\mathscr{S}_{i A B j}\left(\Omega_{1}\right)\right\}\left\langle\dot{F}_{j B}^{*}\right\rangle_{W}
$$

where $\mathscr{S}\left(\Omega_{1}\right)$ and $\mathscr{S}\left(\Omega_{2}\right)$ are the generalized Eshelby tensors corresponding to the ellipsoidal domains $\Omega_{1}$ and $\Omega_{2}$, respectively. The average stress rate in $\Omega_{2}-\Omega_{1}$ is given by

$$
\left\langle\dot{\mathbf{S}}^{N}\right\rangle_{\Omega_{2}-\Omega_{1}}=\frac{W}{\Omega_{2}-\Omega_{1}} \mathscr{F}:\left\{\mathscr{S}\left(\Omega_{2}\right)-\mathscr{S}\left(\Omega_{1}\right)\right\}:\left\langle\dot{\mathbf{F}}^{*}\right\rangle_{W} .
$$

Eqs. (79a)-(79c) are the finite-deformation version of the so-called Tanaka-Mori result (Tanaka and Mori, 1972; see also, Nemat-Nasser and Hori, 1993, Section 11).

The generalized Eshelby tensor depends on the matrix properties, defined by $\mathscr{F}$, but not on the properties of $\Omega$. It, however, depends on both the shape and the orientation of the ellipsoid. Moreover, only the ratios of the axes enter the components of this tensor in a coordinate system coincident with the directions of the principal axes of the ellipsoid. Therefore, if the two ellipsoidal regions, $\Omega_{1}$ and $\Omega_{2}$, have the same shape and orientation, i.e., if the corresponding principal axes have common ratios and directions, and since both 
are embedded in the same unbounded domain, then

$\mathscr{S}\left(\Omega_{2}\right)=\mathscr{S}\left(\Omega_{1}\right)$.

Hence, the average velocity gradient and the average nominal stress rate in $\Omega_{2}-\Omega_{1}$ vanish,

$\langle\dot{\mathbf{F}}\rangle_{\Omega_{2}-\Omega_{1}}=\mathbf{0}$,

$\left\langle\dot{\mathbf{S}}^{N}\right\rangle_{\Omega_{2}-\Omega_{1}}=\mathbf{0}$.

These exact results hold for any $W$ of any arbitrary shape, containing any arbitrary eigenvelocity gradient, $\dot{\mathbf{F}}^{*}(\mathbf{X})$.

\subsection{Generalized phase-transformation problem}

Here we generalize to finite deformations and rotations, some exact averaging results recently presented by Nemat-Nasser and Hori $(1993,1995)$ for linearly elastic solids. These authors seek to obtain the average strains and stresses in an ellipsoidal region $V$ within an unbounded uniform linearly elastic solid $B$ of elasticity tensor $\mathbf{C}$, when $V$ undergoes phase transformation in the following manner: (1) an ellipsoidal subregion $\Omega$ of $V$ undergoes transformation corresponding to variable eigenstrains $\boldsymbol{\varepsilon}^{* 1}(\mathbf{x})$; and (2) the remaining part of $V, \Gamma \equiv V-\Omega$, undergoes transformation with uniform eigenstrains $\boldsymbol{\varepsilon}^{* 2}$.

Consider a finitely deformed solid of any constitutive property which can be represented by Eqs. (30a)-(30c) in terms of the nominal stress rate, $\dot{\mathbf{S}}^{N}$, and velocity gradient, $\dot{\mathbf{F}}$. This is shown in Fig. 4. Eigenvelocity gradients (variable), $\dot{\mathbf{F}}^{* 1}(\mathbf{X})$, are prescribed in $\Omega$, and constant eigenvelocity gradients, $\dot{\mathbf{F}}^{* 2}$, are prescribed in the remaining part, $V-\Omega$. Ellipsoids $\Omega$ and $V$ need be neither coaxial nor similar.

Although the resulting velocity gradient and nominal stress rate fields, $\dot{\mathbf{F}}$ and $\dot{\mathbf{S}}^{N}$, in general are not constant in $V$ and $\Omega$, the average velocity gradient and nominal stress rate in $\Omega$ are exactly given by

$$
\begin{aligned}
\langle\dot{\mathbf{F}}\rangle_{\Omega}= & \mathscr{S}^{\Omega}:\left\langle\dot{\mathbf{F}}^{* 1}\right\rangle_{\Omega}+\left(\mathscr{S}^{V}-\mathscr{S}^{\Omega}\right): \dot{\mathbf{F}}^{* 2}, \\
\left\langle\dot{\mathbf{S}}^{N}\right\rangle_{\Omega}= & \mathscr{\mathscr { F }}:\left(\mathscr{S}^{\Omega}-\mathbf{1}^{(4)}\right):\left\langle\dot{\mathbf{F}}^{* 1}\right\rangle_{\Omega} \\
& +\mathscr{\mathscr { F }}:\left(\mathscr{S}^{V}-\mathscr{S}^{\Omega}\right): \dot{\mathbf{F}}^{* 2},
\end{aligned}
$$

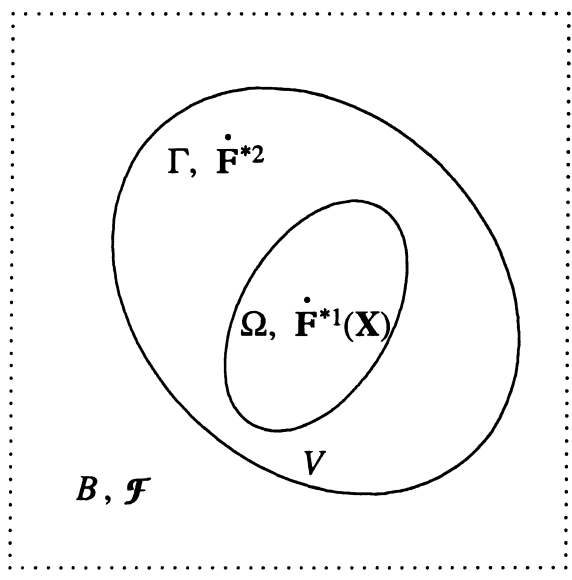

Fig. 4. An unbounded uniform solid $B$ contains two ellipsoidal regions, $V$ and $\Omega(\Omega \subset V)$, with eigenvelocity gradients $\dot{\mathbf{F}}^{* 1}(\mathbf{X})$ in $\Omega$ and uniform eigenvelocity gradients $\dot{\mathbf{F}}^{* 2}$ in $\Gamma=V-\Omega$.

where $\mathscr{S}^{V}$ and $\mathscr{S}^{\Omega}$ are the generalized Eshelby's tensors for ellipsoids $V$ and $\Omega$, respectively, as defined in Section 4.5. Similarly, the average velocity gradient and nominal stress rate over $V$ are exactly given by

$$
\begin{aligned}
& \langle\dot{\mathbf{F}}\rangle_{V}=\mathscr{S}^{V}:\left\{f\left\langle\dot{\mathbf{F}}^{* 1}\right\rangle_{\Omega}+(1-f) \dot{\mathbf{F}}^{* 2}\right\}, \\
& \left\langle\dot{\mathbf{S}}^{N}\right\rangle_{V}=\mathscr{F}:\left(\mathscr{S}^{V}-\mathbf{1}^{(4)}\right):\left\{f\left\langle\dot{\mathbf{F}}^{* 1}\right\rangle_{\Omega}+(1-f) \dot{\mathbf{F}}^{* 2}\right\},
\end{aligned}
$$

where $f$ is the volume fraction of $\Omega$ in $V, f=\Omega / V$. Finally, in view of Eqs. (81a)-(81d), the average velocity gradient and nominal stress rate over the annulus $\mathrm{G}$ are given by

$$
\begin{aligned}
&\langle\dot{\mathbf{F}}\rangle_{\Gamma}=\mathscr{S}^{V}: \dot{\mathbf{F}}^{* 2}+\frac{f}{1-f}\left(\mathscr{S}^{V}-\mathscr{S}^{\Omega}\right):\left(\left\langle\dot{\mathbf{F}}^{* 1}\right\rangle_{\Omega}-\dot{\mathbf{F}}^{* 2}\right),(81 \mathrm{e}) \\
&\left\langle\dot{\mathbf{S}}^{N}\right\rangle_{\Gamma}=\mathscr{\mathscr { F }}:\left(\mathscr{S}^{V}-\mathbf{1}^{(4)}\right): \dot{\mathbf{F}}^{* 2} \\
& \quad+\frac{f}{1-f} \mathscr{\mathscr { F }}:\left(\mathscr{S}^{V}-\mathscr{S}^{\Omega}\right):\left(\left\langle\dot{\mathbf{F}}^{* 1}\right\rangle_{\Omega}-\dot{\mathbf{F}}^{* 2}\right) .
\end{aligned}
$$

Again, these equations are exact.

The proof of Eqs. (81a)-(81f) directly follows from the result presented in the preceding section. 
The volume average of the velocity gradient produced by $\dot{\mathbf{F}}^{* 1}(\mathbf{X})$ in $\Omega$ is

$\left\langle\left(\text { velocity gradient due to } \dot{\mathbf{F}}^{* 1}\right)\right\rangle_{D}$

$$
= \begin{cases}\mathscr{S}^{\Omega}:\left\langle\dot{\mathbf{F}}^{* 1}\right\rangle_{\Omega} & D=\Omega \\ f \mathscr{S}^{V}:\left\langle\dot{\mathbf{F}}^{* 1}\right\rangle_{\Omega} & D=\mathrm{V}\end{cases}
$$

and the volume average of the corresponding nominal stress rate field is

$\left\langle\left(\text { nominal stress rate due to } \dot{\mathbf{F}}^{* 1}\right)\right\rangle_{D}$

$$
= \begin{cases}\mathscr{F}:\left(\mathscr{S}^{V}-\mathbf{1}^{(4)}\right):\left\langle\dot{\mathbf{F}}^{* 1}\right\rangle_{\Omega} & D=\Omega \\ f \mathscr{F}:\left(\mathscr{S}^{V}-\mathbf{1}^{(4)}\right):\left\langle\dot{\mathbf{F}}^{* 1}\right\rangle_{\Omega} & D=V .\end{cases}
$$

Fields due to constant $\dot{\mathbf{F}}^{* 2}$ in $\Gamma$ can be obtained by superposing the fields due to (constant) $-\dot{\mathbf{F}}^{* 2}$ distributed over the entire $\Omega$ and the fields due to (constant) $\dot{\mathbf{F}}^{* 2}$ distributed over the entire $V$. Hence, the volume average of the velocity gradient and nominal stress rate fields produced by $\dot{\mathbf{F}}^{* 2}$ in $\Gamma$ are computed by applying expressions (75a) and (75b) and (76a) and (76b) separately to the fields due to $-\dot{\mathbf{F}}^{* 2}$ in $\Omega$ and $-\dot{\mathbf{F}}^{* 2}$ in $V$, i.e.,

$\left\langle\left(\text { velocity gradient due to } \dot{\mathbf{F}}^{* 2}\right)\right\rangle_{D}$

$$
= \begin{cases}\left(\mathscr{S}^{V}-\mathscr{S}^{V}\right): \dot{\mathbf{F}}^{* 2} & D=\Omega \\ (1-f) \mathscr{S}^{V}: \dot{\mathbf{F}}^{* 2} & D=V,\end{cases}
$$

$\left\langle\left(\text { nominal stress rate to } \dot{\mathbf{F}}^{* 2}\right)\right\rangle_{D}$

$$
= \begin{cases}\mathscr{F}:\left(\mathscr{S}^{V}-\mathscr{S}^{\Omega}\right): \dot{\mathbf{F}}^{* 2} & D=\Omega \\ (1-f) \mathscr{P}:\left(\mathscr{S}^{V}-\mathbf{1}^{(4)}\right): \dot{\mathbf{F}}^{* 2} & D=V .\end{cases}
$$

These expressions are the generalized version of Eqs. $(10.4 .17 \mathrm{c}, \mathrm{d})$ of Nemat-Nasser and Hori (1993). ${ }^{19}$ The volume averages of $\dot{\mathbf{F}}$ and $\dot{\mathbf{S}}^{N}$ taken over $\Omega$ and $V$, Eqs. (81a)-(81d), are obtained directly from Eqs. (82a)-(82d).

\footnotetext{
${ }^{19}$ In Nemat-Nasser and Hori (1993), there are typographical errors (superscripts) in their Eqs. (10.4.17c,d), page 349. Their (10.4.17d) should read:

$\left\langle\left(\text { stress due to } \boldsymbol{\varepsilon}^{* 2}\right)\right\rangle_{D}= \begin{cases}\mathbf{C}:\left(\mathbf{S}^{V}-\mathbf{S}^{\Omega}\right): \boldsymbol{\varepsilon}^{* 2} & D=\Omega \\ (1-f) \mathbf{C}:\left(\mathbf{S}^{V}-\mathbf{1}^{(4 s)}\right): \boldsymbol{\varepsilon}^{* 2} & D=V .\end{cases}$
}

\subsection{A nested sequence of transforming ellipsoidal regions}

The above results can be generalized to the case when $V$ consists of a series of annulus subregions, i.e., a nested sequence of ellipsoidal regions, where, in each of the annuli, a distinct but constant eigenvelocity gradient is prescribed. As in the case of linear elasticity (Nemat-Nasser and Hori, 1993, 1995), the resulting average field quantities can be computed exactly, using the procedure outlined in the preceding section.

To show this, consider a nested series of ellipsoidal regions, $\Omega_{\alpha}(\alpha=1,2, \ldots, m)$, with $\Omega_{m} \equiv V$, which satisfy $\Omega_{1} \subset \Omega_{2} \subset \ldots \subset \Omega_{m}$, and denote the annulus between $\Omega_{\alpha}$ and $\Omega_{\alpha-1}$ by $\Gamma_{\alpha} \equiv \Omega_{\alpha}-\Omega_{\alpha-1}$ $(\alpha=2,3, \ldots, m)$; see Fig. 5 . These ellipsoids need be neither coaxial nor similar. Now, consider the following distribution of eigenvelocity gradients:

$\dot{\mathbf{F}}^{*}(\mathbf{X})=H\left(\mathbf{X} ; \Omega_{1}\right) \dot{\mathbf{F}}^{* 1}(\mathbf{X})+\sum_{\alpha=2}^{m} H\left(\mathbf{X} ; \Gamma_{\alpha}\right) \dot{\mathbf{F}}^{* \alpha}$,

where each $\dot{\mathbf{F}}^{* \alpha}(\alpha=2,3, \ldots, m)$ is constant, and $H(\mathbf{X} ; D)$ is the Heaviside step function, taking on the value 1 when $\mathbf{X}$ is in $D$, and 0 otherwise; note that $\dot{\mathbf{F}}^{* 1}(\mathbf{X})$ need not be constant, and that each annulus has a different but constant eigenvelocity gradient. The resulting velocity gradient and nominal stress rate fields are denoted by $\dot{\mathbf{F}}$ and $\dot{\mathbf{S}}^{N}$, respectively.

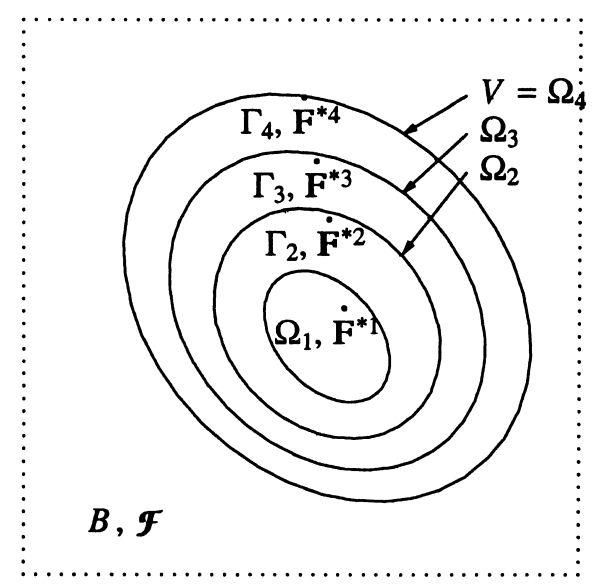

Fig. 5. A nested sequence of four inclusions embedded in infinite domain $B ; \quad \Omega_{\alpha}$ with eigenvelocity gradients $\dot{\mathbf{F}}^{* \alpha}$ $(\alpha=1,2,3,4) ; B$ with pseudo-modulus tensor $\mathscr{\mathscr { Y }}$. 
Apply expressions $(75 \mathrm{a}, \mathrm{b})$ and $(76 \mathrm{a}, \mathrm{b})$ to the fields produced by $\dot{\mathbf{F}}^{* 1}(\mathbf{X})$ in $\Omega_{1}$. The volume average of the resulting velocity gradient field over any $\Omega_{\alpha} \supseteq \Omega_{1}$ then is

$\left\langle\text { velocity gradient due to } \dot{\mathbf{F}}^{* 1}\right\rangle_{\alpha}=\frac{\Omega_{1}}{\Omega_{\alpha}} \mathscr{S}^{\alpha}:\left\langle\dot{\mathbf{F}}^{* 1}\right\rangle_{1}$

where $\alpha$ is not summed, $\mathscr{S}^{\alpha}$ is the generalized Eshelby tensor for $\Omega_{\alpha}$, and subscript $\alpha$ or 1 on \langle\rangle emphasizes that the volume average is taken over $\Omega_{\alpha}$ or $\Omega_{1}$. The velocity gradient field due to $\dot{\mathbf{F}}^{* \beta}$ in $\Gamma_{\beta}(\beta=2,3, \ldots, m)$ is obtained by superposing the velocity gradient field due to $-\dot{\mathbf{F}}^{* \beta}$ distributed in $\Omega_{\beta-1}$, and the velocity gradient field due to $\dot{\mathbf{F}}^{* \beta}$ distributed in $\Omega_{\beta}$. Hence, the average velocity gradient over $\Omega_{\alpha}$ due to $\dot{\mathbf{F}}^{* \beta}$ is

$\left\langle\text { velocity gradient due to } \dot{\mathbf{F}}^{* \beta}\right\rangle_{\alpha}$

$$
= \begin{cases}\left(\mathscr{S}^{\beta}-\mathscr{S}^{\beta-1}\right): \dot{\mathbf{F}}^{* \beta} & \Omega_{\alpha} \subset \Omega_{\beta} \\ \left\{\left(\Omega_{\beta}-\Omega_{\beta-1}\right) / \Omega_{\alpha}\right\} \mathscr{S}^{\alpha}: \dot{\mathbf{F}}^{* \beta} & \Omega_{\alpha} \supseteq \Omega_{\beta} \\ (\beta \text { not summed }) . & \end{cases}
$$

Since all subregions have the same pseudo-modulus tensor, $\mathscr{\mathscr { F }}$, the resulting average nominal stress rates are obtained directly from the corresponding average (velocity gradient - eigenvelocity gradient).

The volume average of the velocity gradient field $\dot{\mathbf{F}}$, over $\Omega_{\alpha}$ is obtained by superposition of Eq. (84a) and Eq. (84b) for $\beta=2,3, \ldots, m$, and then the volume average of $\dot{\mathbf{F}}$ over annular region $\Gamma_{\alpha}$ is computed. This leads to

$$
\langle\dot{\mathbf{F}}\rangle_{1}=\mathscr{S}^{1}:\left\langle\dot{\mathbf{F}}^{* 1}\right\rangle_{1}+\sum_{\beta=2}^{m}\left(\mathscr{S}^{\beta}-\mathscr{S}^{\beta-1}\right): \dot{\mathbf{F}}^{* \beta}
$$

and

$$
\begin{aligned}
\langle\dot{\mathbf{F}}\rangle_{1}^{\prime}= & \frac{1}{F_{\alpha}-F_{\alpha-1}}\left(\mathscr{S}^{\alpha}-\mathscr{S}^{\alpha-1}\right):\left\{F_{1}\left\langle\dot{\mathbf{F}}^{* 1}\right\rangle_{1}\right. \\
& \left.+\sum_{\beta=2}^{m}\left(F_{\beta}-F_{\beta-1}\right) \dot{\mathbf{F}}^{* \beta}\right\} \\
& +\frac{1}{F_{\alpha}-F_{\alpha-1}}\left\{\left(F_{\alpha}-2 F_{\alpha-1}\right) \mathscr{S}^{\alpha}\right. \\
& \left.+F_{\alpha-1} \mathscr{S}^{\alpha-1}\right\}: \dot{\mathbf{F}}^{* \alpha}+\sum_{\beta=\alpha+1}^{m}\left(\mathscr{S}^{\beta}-\mathscr{S}^{\beta-1}\right): \dot{\mathbf{F}}^{* \beta}
\end{aligned}
$$

for $\alpha=2,3, \ldots, m$, where \langle\rangle$_{\alpha}^{\prime}$ denotes the volume average taken over the annular region $\Gamma_{\alpha}$, and $F_{\alpha}$ is the volume fraction of $\Omega_{\alpha}$ relative to $\Omega_{m} \equiv V$, i.e.,

\langle\rangle$_{\alpha}^{\prime} \equiv \frac{1}{F_{\alpha}-F_{\alpha-1}}\left(F_{\alpha}\langle\rangle_{\alpha}-F_{\alpha-1}\langle\rangle_{\alpha-1}\right)$,

$F_{\alpha}=\frac{\Omega_{\alpha}}{V}$.

The corresponding average nominal stress rates are computed in the same manner, and are expressed in terms of $\langle\dot{\mathbf{F}}\rangle$, as

$\left\langle\dot{\mathbf{S}}^{N}\right\rangle_{1}=\mathscr{\mathscr { F }}:\left(\langle\dot{\mathbf{F}}\rangle_{1}-\left\langle\dot{\mathbf{F}}^{* 1}\right\rangle_{1}\right)$,

$\left\langle\dot{\mathbf{S}}^{N}\right\rangle_{\alpha}^{\prime}=\mathscr{F}:\left(\langle\dot{\mathbf{F}}\rangle_{\alpha}-\dot{\mathbf{F}}^{* \alpha}\right)$,

for $\alpha=2,3, \ldots, m$. In particular, if all $\Omega_{\alpha}^{\prime}$ 's are similar and coaxial, then, Eq. (85a) and Eq. (85b) become

$$
\begin{aligned}
\langle\dot{\mathbf{F}}\rangle_{1} & =\mathscr{S}:\left\langle\dot{\mathbf{F}}_{1}^{* 1}\right\rangle_{1}, \\
\langle\dot{\mathbf{F}}\rangle_{\alpha}^{\prime} & =\mathscr{S}: \dot{\mathbf{F}}^{* \alpha} .
\end{aligned}
$$

for $\alpha=2,3, \ldots, m$, where $\mathscr{S}$ is the generalized Eshelby tensor common to all $\Omega_{\alpha}$ 's. ${ }^{20}$

\section{Calculation of Green's function}

As pointed out in Section 4.2, the Green function, $G_{j m}^{\infty}(\mathbf{X})$, for an infinitely extended homogeneous domain, $V^{\infty}$, is the vector-valued fundamental solution of the operator

$\mathscr{L}_{i j} \equiv \mathscr{F}{ }_{A i B j} \frac{\partial^{2}}{\partial X_{A} \partial X_{B}}$

defined by the following boundary-value problem:

$\mathscr{F}_{A i B j} G_{j m, A B}^{\infty}(\mathbf{X})+\delta(\mathbf{X}) \delta_{i m}=0 \quad \mathbf{X}$ in $V^{\infty}$,

where $\delta(\mathbf{X})$ is the delta function, and

$G_{j m}^{\infty}(\mathbf{X}) \rightarrow 0 \quad$ as $|\mathbf{X}| \rightarrow \infty$.

Since the matrix of the pseudo-modulus tensor $\mathscr{\mathscr { F }}$, has diagonal symmetry, i.e., since $\mathscr{F}_{A i B j}=\mathscr{F}_{B j A i}$, it follows that the operator $\mathscr{L}_{i j}$ is symmetric,

\footnotetext{
${ }^{20}$ Note that in (85b), the first summation is omitted for $\alpha=2$, and the second summation is omitted for $\alpha=m$.
} 
$\mathscr{L}_{i j} \equiv \mathscr{L}_{j i} \quad i, j,=1,2,3$

As shown in Section 4.2, this symmetry leads to the symmetry of the Green function, $G_{j m}^{\infty}(\mathbf{X})=G_{m j}^{\infty}(\mathbf{X})$. It thus follows that only the following combination of the components of $\mathscr{F}$ enters the expression of the Green function:

$\hat{\mathscr{F}}_{A i B j}=\frac{1}{4}\left(\mathscr{F}_{A i B j}+\mathscr{\mathscr { F }}_{B i A j}+\mathscr{\mathscr { F }}_{A j B i}+\mathscr{\mathscr { F }}_{B j A i}\right)$.

The tensor $\hat{\mathscr{F}}_{A i B j}$, in general, has a total of 36 distinct components.

\subsection{Strong ellipticity and Green's function}

The operator $\mathscr{L}_{i j}$ is called strongly elliptic if

$\mathscr{F}_{A i B j} \xi_{A} \xi_{B} n_{i} n_{j}>0 \quad A, B, i, j=1,2,3$,

for any nonzero pair of vectors, $\boldsymbol{\xi}$ and $\mathbf{n}$, with rectangular Cartesian components, $\xi_{A}$ and $n_{i}$, $A, i=1,2,3$. The existence of strong ellipticity precludes the inception of strain localization in the form of discontinuity surfaces, across which certain components of the velocity gradient undergo finite jumps; Hadamard (1903), Thomas (1956, 1958), Hill (1961, 1962), Rice (1977), and Iwakuma and Nemat-Nasser (1984).

Indeed, suppose $\mathbf{N}$ is a unit vector normal to such a surface, say, $\Sigma$, at point $\mathbf{X}$; see Fig. 6 . Since the traction rates must remain continuous across $\Sigma$, it follows that the jump in the traction rates, defined by $[\dot{\mathbf{T}}](\mathbf{X})$, must be zero. This jump is given in terms of the nominal stress rate and the velocity gradient, by

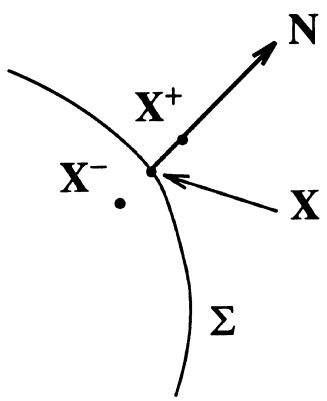

Fig. 6. A surface of discontinuity $\Sigma$, across which certain components of $\dot{\mathbf{F}}$ may be discontinuous.

$$
\begin{aligned}
{[\dot{\mathbf{T}}](\mathbf{X}) } & =\lim _{\mathbf{X}^{-}, \mathbf{X}^{+} \rightarrow \mathbf{X}} \mathbf{N} \cdot\left\{\dot{\mathbf{S}}^{N}\left(\mathbf{X}^{+}\right)-\dot{\mathbf{S}}^{N}\left(\mathbf{X}^{-}\right)\right\} \\
& =\mathbf{N} \cdot \mathscr{F}:[\dot{\mathbf{F}}](\mathbf{X})
\end{aligned}
$$

where

$[\dot{\mathbf{F}}](\mathbf{X})=\lim _{\mathbf{X}^{-}, \mathbf{X}^{+} \rightarrow \mathbf{X}}\left\{\dot{\mathbf{F}}\left(\mathbf{X}^{+}\right)-\dot{\mathbf{F}}\left(\mathbf{X}^{-}\right)\right\}=\boldsymbol{\eta} \otimes \mathbf{N}$.

Here, $\boldsymbol{\eta}$ is the vector-valued amplitude of the jump. In component form, these expressions become

$$
\begin{aligned}
{\left[\dot{T}_{i}\right](\mathbf{X}) } & =\lim _{\mathbf{X}^{-}, \mathbf{X}^{+} \rightarrow \mathbf{X}} N_{A}\left\{\dot{S}_{A j}^{N}\left(\mathbf{X}^{+}\right)-\dot{S}_{A i}^{N}\left(\mathbf{X}^{-}\right)\right\} \\
& =N_{A} \mathscr{F}_{A i B j}\left[\dot{F}_{j B}\right](\mathbf{X}),
\end{aligned}
$$

where

$\left[\dot{F}_{i A}\right](\mathbf{X})=\lim _{\mathbf{X}^{-}, \mathbf{X}^{+} \rightarrow \mathbf{X}}\left\{\dot{F}_{i A}\left(\mathbf{X}^{+}\right)-\dot{F}_{i A}\left(\mathbf{X}^{-}\right)\right\}=\eta_{i} N_{A}$.

From Eqs. $(91 \mathrm{a}, \mathrm{b})$ and Eqs. $(92 \mathrm{a}, \mathrm{b})$, the condition for the continuity of the traction rates across $\Sigma$ becomes (Hill, 1962; Rice, 1977; and Iwakuma and Nemat-Nasser, 1984),

$[\dot{\mathbf{T}}](\mathbf{X})=\mathbf{N} \cdot \mathscr{F}:(\boldsymbol{\eta} \otimes \mathbf{N})=\mathbf{0}$

or, in components,

$\left[\dot{T}_{i}\right](\mathbf{X})=\left(\mathscr{F}_{A i B j} N_{A} N_{B}\right) \eta_{j}=0$.

For nontrivial solutions, the determinant of the coefficients of $\boldsymbol{\eta}$ in the set of three homogeneous linear Eqs. (93a,b) must vanish, leading to the following characteristic equation for the operator $\mathscr{L}_{i j}$ :

$\mathscr{D}(\mathbf{N}) \equiv \operatorname{det}\left|\mathscr{F}_{A i B j} N_{A} N_{B}\right|=0$.

The inception of discontinuous solutions is possible if Eq. (94) admits real-valued roots for the vector $\mathbf{N}$. If $\mathbf{N}^{\mathrm{I}}$ is one such root, then from Eqs. $(93 \mathrm{a}, \mathrm{b})$ the corresponding amplitude, $\boldsymbol{\eta}^{I}$, is computed, leading to

$(\boldsymbol{\eta} \otimes \mathbf{N})^{I}: \mathscr{F}:(\boldsymbol{\eta} \otimes \mathbf{N})^{I}=\mathscr{F}_{A i B j} N_{A}^{I} N_{B}^{I} \eta_{i}^{I} \eta_{j}^{I}=0$.

This is precluded by the strong ellipticity condition (90). Thus, the strong ellipticity guarantees complex-valued solutions, $\mathbf{N}$, for the characteristic Eq. (94). Since the coefficients of this sextic equation are real, the roots occur as pairs of complex conjugates. In such a case, Eqs. $(89 \mathrm{a}-\mathrm{c})$ yields a real-valued Green function. 


\subsection{Green's function}

The Green function can be obtained by applying the Fourier transformation to Eqs. (89a-c). Denote the Fourier transform of the Green function $\mathbf{G}^{\infty}(\mathbf{X})$ by $\hat{\mathbf{G}}^{\infty}(\boldsymbol{\xi})$, i.e.,

$G_{i j}^{\infty}(\mathbf{X})=\frac{1}{(2 \pi)^{3}} \int_{-\infty}^{\infty} \hat{G}_{i j}^{\infty}(\boldsymbol{\xi}) \exp (i \xi \cdot \mathbf{X}) \mathrm{d} V_{\xi}$,

where $\mathrm{d} V_{\xi}$ is the elementary volume element in the $\xi$-space. Hence, the Fourier transform of $G_{i j, A B}^{\infty}(\mathbf{X})$ is $-\hat{G}_{i j}^{\infty}(\xi) \xi_{A} \xi_{B}$. Since

$\delta(\mathbf{X})=\frac{1}{(2 \pi)^{3}} \int_{-\infty}^{\infty} \exp (i \xi \cdot \mathbf{X}) \mathrm{d} V_{\xi}$,

the Fourier transform of Eq. (89a) results in

$K_{i j}(\xi) \hat{G}_{j m}^{\infty}(\xi)=\delta_{i m}$,

where

$K_{i j}(\xi)=\mathscr{F}_{A i B j} \xi_{A} \xi_{B}$.

With strong ellipticity, matrix $K(\xi)$ admits an inverse for all values of $\xi$. Hence, the Fourier transform of the Green function becomes

$\hat{G}_{i j}^{\infty}(\xi)=K_{i j}^{-1}(\xi)=\frac{N_{i j}(\xi)}{\mathscr{D}(\xi)}$,

where

$$
\begin{aligned}
N_{i j}(\xi) & =\frac{1}{2} e_{i k m} e_{j l n} K_{k l}(\xi) K_{m n}(\xi) \\
& =\operatorname{cofactor}\left(K_{i j}(\xi)\right), \\
\mathscr{D}(\xi) & =\frac{1}{6} e_{i k m} e_{j l n} K_{i j}(\xi) K_{k l}(\xi) K_{m n}(\xi) \\
& =\operatorname{det}\left|K_{i j}(\xi)\right|,
\end{aligned}
$$

and $e_{i j k}$ is the permutation symbol.

Eqs. (96a) and (98a) show the relation between the structure of the Green function and the possibility of a loss of stability of the uniformly stressed homogeneous solid, because the nature of the roots of $\mathscr{D}(\xi)=0$ defines the ellipticity, parabolicity, or hyperbolicity of the operator Eq. (98a). As shown in the preceding section, the necessary conditions for the possibility of jumps of certain components of the velocity gradient across a discontinuity surface coincide with the conditions for the existence of the real roots of $\mathscr{D}(\xi)=0$, which then precludes the existence of a real-valued Green function, since, in that case, the operator Eq. (98a) will no longer be elliptic; Iwakuma and Nemat-Nasser (1984).

To evaluate Eq. (96a), set ${ }^{21}$

$\xi=(\xi \cdot \xi)^{1 / 2}$,

$\bar{\xi}=\xi / \xi$,

and rewrite it as follows:

$$
\begin{aligned}
& G_{i j}^{\infty}(\mathbf{X}) \\
& =\frac{1}{(2 \pi)^{3}} \int_{0}^{\infty} \mathrm{d} \xi \int_{S(\bar{\xi})} \frac{N_{i j}(\bar{\xi})}{\mathscr{D}(\bar{\xi})} \exp \{i \xi \bar{\xi} \cdot \mathbf{X}\} \mathrm{d} S(\bar{\xi}),
\end{aligned}
$$

where $\mathrm{d} S(\xi)$ is an elementary surface of a unit sphere, $S(\bar{\xi})$, in the $\bar{\xi}$-space. In deriving Eq. (100a), the property that $N_{i j}(\xi)$ and $\mathscr{D}(\xi)$ are fourth- and sixth-degree polynomials in $\xi$ is used. Since the delta function satisfies

$\delta(x)=\frac{1}{2 \pi} \int_{-\infty}^{\infty} \exp \{i \xi x\} \mathrm{d} \xi$,

Eq. (100a) can be written as

$G_{i j}^{\infty}(\mathbf{X})=\frac{1}{8 \pi^{2}} \int_{S(\bar{\xi})} \frac{N_{i j}(\boldsymbol{\xi})}{\mathscr{D}(\xi)} \delta(\bar{\xi} \cdot \mathbf{X}) \mathrm{d} S(\bar{\xi})$.

\subsection{Generalized Eshelby tensor and its conjugate}

For the present application, substitute Eqs. (69e) and (69f) into Eq. (73a) and use Eq. (96a) to arrive at

$$
\begin{aligned}
\mathscr{P}_{i A j B}(\mathbf{X} ; \Omega)= & \frac{-1}{(2 \pi)^{3}} \frac{\partial^{2}}{\partial X_{A} \partial X_{B}} \int_{W} \mathrm{~d} V_{\mathbf{Y}} \int_{-\infty}^{\infty} \hat{G}_{i j}^{\infty}(\boldsymbol{\xi}) \\
& \times \exp \{i \boldsymbol{\xi} \cdot(\mathbf{X}-\mathbf{Y})\} \mathrm{d} V_{\xi} .
\end{aligned}
$$

Now, introduce the following coordinate transformation:

\footnotetext{
${ }^{21}$ See Kröner (1953), Kneer (1965), Willis (1964), Indenbom and Orlov (1968), Kinoshita and Mura (1971) and Mura (1987).
} 
$\zeta_{A}=a_{A} \xi_{A}, \quad \bar{X}_{A}=X_{A} / a_{A}, \quad \bar{Y}_{A}=Y_{A} / a_{A}$,

$A=1,2,3,(A$ not summed $)$,

where $a_{A}, A=1,2,3$, are constants. An ellipsoidal $\Omega$ whose principal axes are parallel to the global coordinate axes $X_{A}$, with length $2 a_{i}, i=1,2,3$, is then transformed into a unit sphere $\Omega$ in the $\mathbf{X}$ space. In terms of the coordinates $\overline{\mathbf{X}}, \overline{\mathbf{Y}}$, and $\zeta$, Eq. (102a) reduces to

$$
\begin{aligned}
\mathscr{P}_{i A j B}(\overline{\mathbf{X}} ; \overline{\boldsymbol{\Omega}})= & \frac{-1}{(2 \pi)^{3} a_{A} a_{B}} \frac{\partial^{2}}{\partial \bar{X}_{A} \partial \bar{X}_{B}} \int_{\Omega} \mathrm{d} V_{\overline{\mathbf{Y}}} \int_{-\infty}^{\infty} \hat{G}_{i j}^{\infty *}(\zeta) \\
& \times \exp \{i \zeta \cdot(\overline{\mathbf{X}}-\overline{\mathbf{Y}})\} \mathrm{d} V_{\zeta}, \quad(102 \mathrm{~b})
\end{aligned}
$$

where $A, B$ are not summed,

$$
\begin{aligned}
\hat{G}_{i j}^{\infty *}(\zeta) & =\frac{N_{i j}^{*}(\zeta)}{\mathscr{D}^{*}(\zeta)} \\
N_{i j}(\zeta) & =\frac{1}{2} e_{i k m} e_{j l n} K_{k l}^{*}(\zeta) K_{m n}^{*}(\zeta) \\
& =\operatorname{cofactor}\left(K_{i j}^{*}(\zeta)\right), \\
\mathscr{D}^{*}(\zeta) & =\frac{1}{6} e_{i k m} e_{j l n} K_{i j}^{*}(\zeta) K_{k l}^{*}(\zeta) K_{m n}^{*}(\zeta) \\
& =\operatorname{det}\left|K_{i j}^{*}(\zeta)\right| \\
K_{i j}^{*}(\zeta) & =\mathscr{F}^{*}{ }_{A i B j} \zeta_{A} \zeta_{B}
\end{aligned}
$$

(all repeated indices are summed),

$\mathscr{F}^{*}{ }_{A i B j}=\frac{1}{a_{A} a_{B}} \mathscr{F}_{A i B j} \quad(A, B$ not summed $)$,

and $\mathrm{d} V_{\overline{\mathbf{Y}}}$ and $\mathrm{d} V_{\zeta}$ are elementary areas in the $\overline{\mathbf{Y}}$ - and $\zeta$-space, respectively.

To evaluate Eq. (102b), set

$$
\begin{aligned}
& \zeta=(\zeta \cdot \zeta)^{1 / 2}, \\
& \bar{\zeta}=\zeta / \zeta,
\end{aligned}
$$

and rewrite it as follows:

$$
\begin{aligned}
\mathscr{P}_{i A j B}(\overline{\mathbf{X}} ; \overline{\boldsymbol{\Omega}}) & \\
= & \frac{-1}{(2 \pi)^{3} a_{A} a_{B}} \frac{\partial^{2}}{\partial \bar{X}_{A} \partial \bar{X}_{B}} \int_{\Omega} \mathrm{d} V_{\overline{\mathbf{Y}}} \int_{0}^{\infty} \mathrm{d} \zeta \int_{S(\bar{\zeta})} \hat{G}_{i j}^{\infty}(\bar{\zeta}) \\
& \times \exp (i \zeta \bar{\zeta} \cdot \overline{\mathbf{Z}}) \mathrm{d} S(\bar{\zeta})
\end{aligned}
$$

where $A, B$, are not summed, and $\overline{\mathbf{Z}}=\overline{\mathbf{X}}-\overline{\mathbf{Y}}$, and $\mathrm{d} S(\bar{\zeta})$ is an elementary surface of a unit sphere, $S(\bar{\zeta})$, in the $\bar{\zeta}$-space. In view of Eq. (101), this is further reduced to

$$
\begin{aligned}
& \mathscr{P}_{i A j B}(\overline{\mathbf{X}} ; \overline{\boldsymbol{\Omega}}) \\
& \quad=\frac{-1}{2 \pi^{2} a_{A} a_{B}} \frac{\partial^{2}}{\partial \bar{X}_{A} \partial \bar{X}_{B}} \int_{S(\bar{\zeta})} \hat{G}_{i j}^{\infty^{*}}(\bar{\zeta}) \phi(\bar{\zeta}, \overline{\mathbf{X}}, \bar{\Omega}) \mathrm{d} S(\bar{\zeta}),
\end{aligned}
$$

where $A$ and $B$ are not summed, and

$$
\phi(\bar{\zeta}, \overline{\mathbf{X}} ; \bar{\Omega})=\int_{\bar{\Omega}} \delta(\bar{\zeta} \cdot(\overline{\mathbf{X}}-\overline{\mathbf{Y}})) \mathrm{d} V_{\overline{\mathbf{Y}}}
$$

The argument of the delta function in the integrand of Eq. (106a) is zero only if the vector $\overline{\mathbf{Z}}=$ $\overline{\mathbf{X}}-\overline{\mathbf{Y}}$ is normal to $\bar{\zeta}$. Hence, $\phi(\bar{\zeta}, \overline{\mathbf{X}}, \bar{\Omega})$ is the area of a section cut in $\bar{\Omega}$ by the plane which contains the point $\overline{\mathbf{X}}$ and is normal to $\bar{\zeta}$. For any point $\overline{\mathbf{Y}}$ on this plane, $\bar{\zeta} \cdot(\overline{\mathbf{X}}-\overline{\mathbf{Y}})=0$. Since $\bar{\Omega}$ is a unit sphere, the argument of the delta function in Eq. (106a) vanishes for all $\overline{\mathbf{Y}}$ on a circle with radius $\sqrt{1-(\bar{\zeta} \cdot \overline{\mathbf{X}})^{2}}$; see Fig. 7. To evaluate Eq. (105a), define

$z \equiv \bar{\zeta} \cdot \overline{\mathbf{Y}}$

$z_{0} \equiv \bar{\zeta} \cdot \overline{\mathbf{X}}$

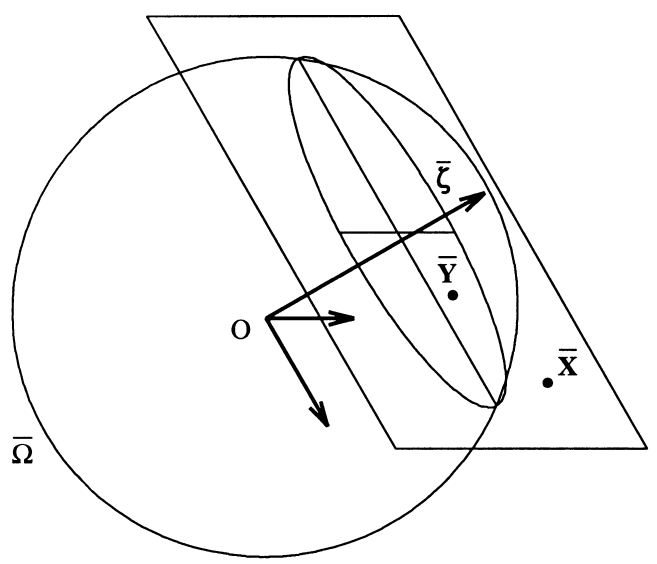

Fig. 7. Area of the section in the unit sphere $\bar{\Omega}$ cut by the plane which contains the point $\overline{\mathbf{X}}$ and is normal to $\bar{\zeta}$. 


$$
R(z) \equiv \sqrt{1-z^{2}}
$$

Then, with respect to a cylindrical coordinate system whose z-axis coincides with $\bar{\zeta}$, Eq. (106a) becomes

$$
\begin{aligned}
\phi^{\bar{\Omega}}(\bar{\zeta}, \overline{\mathbf{X}}) & \equiv \phi(\bar{\zeta}, \overline{\mathbf{X}}, \bar{\Omega}) \\
& =\int_{-1}^{1} \delta\left(z-z_{0}\right) \mathrm{d} z \int_{0}^{R(z)} r \mathrm{~d} r \int_{0}^{2 \pi} \mathrm{d} \theta \\
& = \begin{cases}\pi R^{2}\left(z_{0}\right) & \text { for } z_{0} \leqslant 1 \\
0 & \text { for } z_{0}>1 .\end{cases}
\end{aligned}
$$

Hence,

$$
\phi(\bar{\zeta}, \overline{\mathbf{X}}, \bar{\Omega})= \begin{cases}\pi\left\{1-(\bar{\zeta} \cdot \overline{\mathbf{X}})^{2}\right\} & \text { for } \bar{\zeta} \cdot \overline{\mathbf{X}} \leqslant 1 \\ 0 & \text { for } \bar{\zeta} \cdot \overline{\mathbf{X}}>1\end{cases}
$$

Note that, $\bar{\zeta} \cdot \overline{\mathbf{X}} \leqslant 1$ for all $\overline{\mathbf{X}} \in \overline{\boldsymbol{\Omega}}$. Substitute Eq. (106b) into Eq. (102d) to obtain

$$
\begin{aligned}
\mathscr{P}_{i A j B}^{\Omega} \equiv & \mathscr{P}_{i A j B}(\overline{\mathbf{X}} \in \bar{\Omega} ; \bar{\Omega}) \\
= & \frac{1}{4 \pi a_{A} a_{B}} \int_{S(\bar{\zeta})} \hat{G}_{i j}^{\infty *}(\bar{\zeta}) \bar{\zeta}_{A} \bar{\zeta}_{B} \mathrm{~d} S(\bar{\zeta}), \\
& (A, B \text { not summed }) .
\end{aligned}
$$

Since $\phi$ is a second-order polynomial in $\overline{\mathbf{X}}$, for $\overline{\mathbf{X}} \in \bar{\Omega}, \mathscr{P}^{\Omega}$ is independent of $\overline{\mathbf{X}}$. For $\overline{\mathbf{X}}$ outside of the unit sphere $\bar{\Omega}, \mathscr{P}$ is given by

$$
\begin{aligned}
& \mathscr{P}_{i A j B}(\overline{\mathbf{X}} \notin \overline{\boldsymbol{\Omega}} ; \overline{\boldsymbol{\Omega}}) \\
& \quad=\frac{1}{4 \pi a_{A} a_{B}} \int_{S^{*}(\bar{\zeta})} \hat{G}_{i j}^{\infty *}(\bar{\zeta}) \bar{\zeta}_{A} \bar{\zeta}_{B} \mathrm{~d} S(\bar{\zeta}),
\end{aligned}
$$

where $A$ and $B$ are not summed, and $S^{*}(\bar{\zeta})$ is the subset of the surface area of the unit sphere where $\bar{\zeta} \cdot \overline{\mathbf{X}} \leqslant 1$. Therefore, $\mathscr{P}(\overline{\mathbf{X}} \notin \bar{\Omega}, \bar{\Omega})$ is a function of $\overline{\mathbf{X}}$.

Substitute Eq. (104a) into Eq. (108b) to obtain

$$
\mathscr{P}_{i A j B}^{\Omega}=\frac{1}{4 \pi a_{A} a_{B}} \int_{S(\bar{\zeta})} \frac{N_{i j}^{*}(\bar{\zeta})}{\mathscr{D}^{*}(\bar{\zeta})} \bar{\zeta}_{A} \bar{\zeta}_{B} \mathrm{~d} S(\bar{\zeta}),
$$

( $A, B$ not summed).
To evaluate Eq. (108c), substitute

$\bar{\zeta}_{1}=\cos \phi \cos \theta, \quad \bar{\zeta}_{2}=\cos \phi \sin \theta, \quad \bar{\zeta}_{3}=\sin \phi$,

into it, and obtain

$$
\begin{aligned}
& \mathscr{P}_{i A j B}^{\Omega}=\frac{1}{4 \pi a_{A} a_{B}} \int_{-\pi / 2}^{\pi / 2} \mathrm{~d} \phi \int_{0}^{2 \pi} \frac{N_{i j}^{*}(\bar{\zeta})}{\mathscr{D}^{*}(\bar{\zeta})} \bar{\zeta}_{A} \bar{\zeta}_{B} \mathrm{~d} \theta, \\
& (A, B \text { not summed }) .
\end{aligned}
$$

Now, define

$\mathscr{H}_{i A j B}(\phi) \equiv \frac{1}{2 \pi a_{A} a_{B}} \int_{0}^{2 \pi} \frac{N_{i j}^{*}(\bar{\zeta})}{\mathscr{D}^{*}(\bar{\zeta})} \bar{\zeta}_{A} \bar{\zeta}_{B} \mathrm{~d} \theta$,

( $A, B$ not summed).

and, with $z=e^{i \theta}$, obtain

$\mathscr{H}_{i A j B}(\phi)=\frac{1}{2 \pi i a_{A} a_{B}} \oint_{\gamma} \frac{N_{i j}^{*}(\bar{\zeta})}{z \mathscr{D}^{*}(\bar{\zeta})} \bar{\zeta}_{A} \bar{\zeta}_{B} \mathrm{~d} z$,

( $A, B$ not summed).

where $\gamma$ is the unit circle $|z|=1$ in the complex $z$ plane. Then, Eq. (108d) becomes

$\mathscr{P}_{i A j B}^{\Omega}=\frac{1}{2} \int_{-\pi / 2}^{\pi / 2} \mathscr{H}_{i A j B}(\phi) \mathrm{d} \phi$.

Explicit results emerge for two-dimensional problems whose corresponding Green functions are deduced by the limiting process of $a_{3} \rightarrow \infty$ which renders the integral Eq. (107b) independent of $\phi$. Thus, in two dimensions,

$\mathscr{P}_{i A j B}^{\Omega}=\mathscr{H}_{i A j B}(\phi \rightarrow 0)$,

where the components of $\bar{\zeta}$ are identified with $(\cos \theta, \sin \theta, 0)$. The details are given by Iwakuma and Nemat-Nasser (1984).

\section{Acknowledgements}

This work has been supported by the Army Research Office under contract No. DAAL03-92G-0108 and DAAH04-96-1-0376 with the University of California, San Diego. 


\section{References}

Budiansky, B., 1965. On the elastic moduli of some heterogeneous materials. J. Mech. Phys. Solids 13, 223-227.

Budiansky, B., Wu, T.T., 1962. Theoretical prediction of plastic strains of polycrystals. Proc. Fourth US Natl. Congr. Appl. Mech., ASME, pp. 1175-1185.

Eshelby, J.D., 1957. The determination of the elastic field of an ellipsoidal inclusion, and related problems. Proc. Roy. Soc. London 241A, 376-396.

Hadamard, J., 1903. Lecons sur la Propagations des Ondes et les Equations de l'Hydrodynamique. Ch. 6, Paris, (reprint in New York, 1949) pp. 241-262.

Hardiman, N.J., 1954. Elliptic elastic inclusion in an infinite elastic plate. Q. J. Mech. Appl. Math. 52, 226-230.

Harren, S.V., 1991. The finite deformation of rate dependent polycrystals; I. A self-consistent frame work. J. Mech. Phys. Solids 39, 345-360.

Hashin, Z., 1964. Theory of mechanical behaviour of heterogeneous media. Appl. Mech. Rev. 17, 1-9.

Hashin, Z., 1965. Elasticity of random media. Trans. Soc. Rheol. 9, 381-406.

Hashin, Z., 1983. Analysis of composite materials: A survey. J. Appl. Mech. 50, 481-505.

Havner, K.S., 1982. The theory of finite plastic deformation of crystalline solids. In: Hopkins, H.G., Sewell, M.J. (Eds.), Mechanics of Solids, The Rodney Hill 60th Anniversary Volume, Pergamon Press, Oxford, pp. 265-302.

Havner, K.S., 1992. Finite Plastic Deformation of Crystalline Solids. Cambridge University Press, Cambridge, p. 235.

Hill, R., 1952. The elastic behaviour of a crystalline aggregate. Proc. Phys. Soc. London A 65, 349-354.

Hill, R., 1956. The mechanics of quasi-static plastic deformation in metals. In: Batchelor, G.K., Davies, R.M. (Eds.), Surveys in Mechanics - The G.1. Taylor 70th Anniversary Volume, Cambridge University Press, Cambridge, pp. 7-31.

Hill, R., 1961. Bifurcation and uniqueness in non-linear mechanics of continua. In: Lavrent'ev, M.A. et al. (Eds.), Problems of Continuum Mechanics, SIAM, Philadelphia, pp. 155-164.

Hill, R., 1962. Acceleration waves in solids. J. Mech. Phys. Solids 10, 1-16.

Hill, R., 1965a. Continuum micro-mechanics of elastoplastic polycrystals. J. Mech. Phys. Solids 13, 89-101.

Hill, R., 1965b. A self-consistent mechanics of composite materials. J. Mech. Phys. Solids 13, 213-222.

Hill, R., 1968. On constitutive inequalities for simple materials. J. Mech. Phys. Solids 16, I. 229-242, II. 315-322.

Hill, R., 1970. Constitutive inequalities for isotropic elastic solids under finite strain. Proc. Roy. Soc. London 314A, 457-472.

Hill, R., 1972. On constitutive macro-variables for heterogeneous solids at finite strain. Proc. Roy. Soc. London 326A, 131-147.

Hill, R., 1984. On macroscopic effects of heterogeneity in elastoplastic media at finite strain. Math. Proc. Camb. Phil. Soc. 95, 481-494.
Hill, R., Havner, K.S., 1982. Perspectives in the mechanics of elastoplastic crystals. J. Mech. Phys. Solids 30, 5-22.

Hill, R., Rice, J.R., 1972. Constitutive analysis of elastic-plastic crystals at arbitrary strain. J. Mech. Phys. Solids 20, 401413.

Hori, M., Nemat-Nasser, S., 1994. Double-inclusion model and overall moduli of multi-phase composites. J. Eng. Mater. Tech. 116, 305-309.

Hutchinson, J.W., 1970. Elastic-plastic behavior of polycrystalline metals and composites. Proc. Roy. Soc. London 319A, 247-272.

Indenbom, V.L., Orlov, S.S., 1968. Construction of Green's function in terms of Green's function of lower dimension. J. Appl. Math. Mech. 32, 414 420.

Iwakuma, T., Nemat-Nasser, S., 1983. Composites with periodic microstructure. Comp. Struc. 16, 13-19.

Iwakuma, T., Nemat-Nasser, S., 1984. Finite elastic plastic deformation of polycrystalline metals. Proc. Roy. Soc. London 394A, 87-119.

Kinoshita, N., Mura, T., 1971. Elastic fields of inclusions in anisotropic media. Phys. Status Solidi A 5, 759-768.

Kneer, G., 1965. Uber die Berechnung der Elastizitatsmodulen Vielkristalliner Aggregate mit Textur. Phys. Status Solidi 9, 825-838.

Kröner, E., 1953. Das Fundamentalintegral der anisotropen elastischen Differentialgleichungen. Z. Phys. 136, 402-410.

Kroner, E., 1958. Berechnung der elastischen Konstanten des Vielkristalls aus den Konstanten des Einkristalls. Z. Phys. 151, 504-518.

Kröner, E., 1961. Zur plastischen verformung des Vielkristalls. Acta Met. 9, 155-161.

Kröner, E., 1977. Bounds for effective elastic moduli of disordered materials. J. Mech. Phys. Solids 25, 137-155.

Kröner, E., 1978. Self-consistent scheme and graded disorder in polycrystal elasticity. J. Phys. F 8, 2261-2267.

Mura, T., 1987. Micromechanics of Defects in Solids, 2nd ed. Martinus Nijhoff, Dordrecht, p. 587.

Nemat-Nasser, S., Hori, M., 1993. Micromechanics: OverallProperties of Heterogeneous Solids. Elsevier, Amsterdam.

Nemat-Nasser, S., Hori, M., 1995. Universal Bounds for Overall Properties of Linear and Nonlinear Heterogeneous Solids. Journal of Engineering Materials and Technology 117, 412-432.

Nemat-Nasser, S., Iwakuma, T., Hejazi, M., 1982. On composites with periodic structure. Mech. Mater. I, 239-267.

Nemat-Nasser, S., Obata, M., 1986. Rate-dependent, finite elasto-plastic deformation of polycrystals. Proc. Roy. Soc. London 407A, 343-375.

Rice, J.R., 1971. Inelastic constitutive relations for solids: an internal-variable theory and its application to metal plasticity. J. Mech. Phys. Solids 19, 433-455.

Rice, J.R., 1977. The localization of plastic deformation. In: Koiter, W.T. (Ed.), Theoretical and Applied Mechanics: Proc. 14th IUTAM Congr., North-Holland, pp. 207220. 
Tanaka, K., Mori, T., 1972. Note on volume integrals of the elastic field around an ellipsoidal inclusion. J. Elasticity 2, 199-200.

Thomas, T.Y., 1956. Characteristic surfaces in the PrandtlReuss plasticity theory. J. Rat. Mech. Anal. 5, 251-262.

Thomas, T.Y., 1958. Plastic disturbances whose speed of propagation is less than the velocity of a shear wave. J. Rat. Mech. Anal. 7, 893-900.

Walpole, L.J., 1966a. On bounds for the overall elastic moduli of inhomogeneous systems - I. J. Mech. Phys. Solids 14, 151-162.
Walpole, L.J., 1966b. On bounds for the overall elastic moduli of inhomogeneous systems - II. J. Mech. Phys. Solids 14, 289-301.

Willis, J.R., 1964. Anisotropic elastic inclusion problems. Q. J. Mech. Appl. Math. 17, 157-174.

Willis, J.R., 1977. Bounds and self-consistent estimates for the overall properties of anisotropic composites. J. Mech. Phys. Solids 25, 185-202.

Willis, J.R., 1981. Variational and related methods for the overall properties of composites. Adv. Appl. Mech. 21, 178. 